\title{
Apoptosis reprogramming triggered by splicing inhibitors sensitizes multiple myeloma cells to Venetoclax treatment
}

\author{
Debora Soncini, ${ }^{1}$ Claudia Martinuzzi, ${ }^{1,2}$ Pamela Becherini, ${ }^{1,2}$ Elisa Gelli,, Samantha Ruberti, ${ }^{1}$ \\ Katia Todoerti, ${ }^{3}$ Luca Mastracci, ${ }^{2,4}$ Paola Contini, ${ }^{5}$ Antonia Cagnetta, ${ }^{2}$ Antonella Laudisi, ${ }^{1,2}$ \\ Fabio Guolo, ${ }^{1,2}$ Paola Minetto, ${ }^{2}$ Maurizio Miglino, ${ }^{1,2}$ Sara Aquino, ${ }^{6}$ Riccardo Varaldo, ${ }^{6}$ Daniele \\ Reverberi, ${ }^{7}$ Matteo Formica, ${ }^{2,8}$ Mario Passalacqua, ${ }^{9}$ Alessio Nencioni, ${ }^{2,5}$ Antonino Neri, ${ }^{3,10}$ \\ Mehmet K. Samur, ${ }^{11}$ Nikhil C. Munshi, ${ }^{11}$ Mariateresa Fulciniti, ${ }^{11}$ Roberto M. Lemoli, ${ }^{1,2}$ and \\ Michele Cea., ${ }^{1, \#}$
}

\begin{abstract}
${ }^{1}$ Clinic of Hematology, Department of Internal Medicine (DiMI), University of Genoa, Genoa, Italy; ${ }^{2}$ IRCCS Ospedale Policlinico San Martino, Genoa, Italy; ${ }^{3}$ Hematology, Fondazione Cà Granda IRCCS Policlinico, Milan, Italy; ${ }^{4}$ Department of Integrated Surgical and Diagnostic Sciences, University of Genoa, Genoa, Italy; ${ }^{5}$ Department of Internal Medicine (DiMI), University of Genoa, Genoa, Italy; ${ }^{6} \mathrm{Hematology}$ and Hematopoietic Stem Cell Transplantation Unit, IRCCS Ospedale Policlinico San Martino, Genoa, Italy; ${ }^{7}$ U.O. Molecular Pathology, IRCCS Ospedale Policlinico San Martino, Genoa, Italy; ${ }^{8}$ Department of Surgical Sciences and Integrated Diagnostic (DISC), University of Genoa, Genoa, Italy; ${ }^{\circ}$ Department of Experimental Medicine, University of Genoa, Genoa, Italy; ${ }^{10}$ Department of Oncology and Hemato-oncology, University of Milan, Milan, Italy and "11Jerome Lipper Multiple Myeloma Center, Department of Medical Oncology, Dana Farber Cancer Institute, Harvard Medical School, Boston, MA, USA.
\end{abstract}

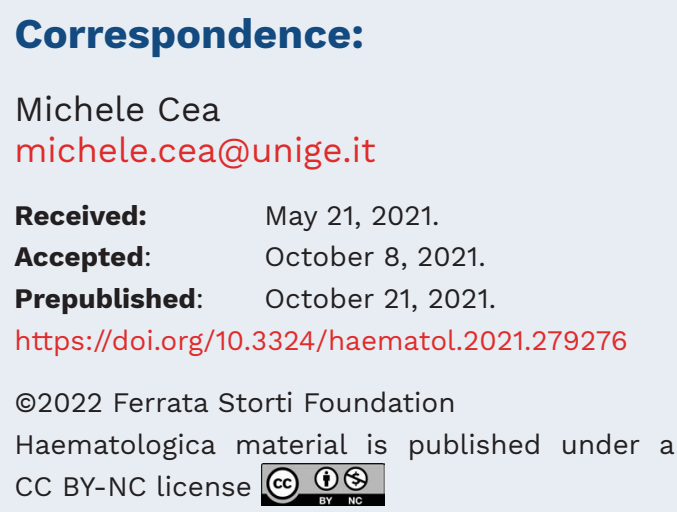

\#RML and MC contributed equally as co-senior authors.

\begin{abstract}
Identification of novel vulnerabilities in the context of therapeutic resistance is emerging as a key challenge for cancer treatment. Recent studies have detected pervasive aberrant splicing in cancer cells, supporting its targeting for novel therapeutic strategies. Here, we evaluated the expression of several spliceosome machinery components in multiple myeloma (MM) cells and the impact of splicing modulation on tumor cell growth and viability. A comprehensive gene expression analysis confirmed the reported deregulation of spliceosome machinery components in MM cells, compared to normal plasma cells from healthy donors, with its pharmacological and genetic modulation resulting in impaired growth and survival of MM cell lines and patient-derived malignant plasma cells. Consistent with this, transcriptomic analysis revealed deregulation of BCL2 family members, including decrease of anti-apoptotic long form of myeloid cell leukemia-1 (MCL1) expression, as crucial for "priming" MM cells for Venetoclax activity in vitro and in vivo, irrespective of $t(11 ; 14)$ status. Overall, our data provide a rationale for supporting the clinical use of splicing modulators as a strategy to reprogram apoptotic dependencies and make all MM patients more vulnerable to BCL2 inhibitors.
\end{abstract}

\section{Introduction}

Multiple myeloma (MM) is a clonal B-cell malignancy characterized by excessive bone marrow plasma cells in association with monoclonal protein., ${ }^{1,2} \mathrm{MM}$ is a heterogeneous disease driven by a large repertoire of molecular abnormalities, which contribute to its diverse clinical behavior. Currently available anti-MM therapies have remarkably improved patient outcome, but resistance is emerging as one of the foremost challenges in the clinical management of this tumor. Therefore, there is an unmet medical need to define biologic mechanisms of drug resistance, both to enhance efficacy of existing treatments and to facilitate the design of novel strategies. ${ }^{3}$ A fundamental feature of $M M$ is its striking genomic instability leading to cancer development and clonal evolution. ${ }^{4,5}$ Consequently, the majority of studies have focused on changes in DNA: unfortunately, the greatest shortcoming of DNA-based approaches is their failure to capture the panoply of RNA editing events. Indeed, the genetic code 
is translated in the cell through the production of a messenger, mRNA, which usually has the same code of the DNA. A percentage of mRNA are "edited", so that although they are made as a faithful copy of the DNA, they are modified afterward to change the products of the genetic code. As a result, pre-mRNA processing by alternative splicing (AS) and/or RNA-specific deaminases, markedly increases the complexity of the human transcriptome. ${ }^{6}$ This process, which is executed in the nucleus by spliceosomes, occurs overwhelmingly in both normal and transformed cells, thus it is the rule rather than the exception. If this process is compromised, the resulting changes in splicing can lead to neoplastic transformation. Indeed, recent studies provide evidence that an abnormally expressed splicing factor machinery as well as its mutations can have oncogenic properties by impacting AS of genes associated with susceptibility and/or progression of cancer. ${ }^{7}$ Despite these intriguing findings, the global pattern of RNA processing in human cancer genomes has not been systematically characterized, and its functional importance and clinical relevance in cancer remains largely unknown. Thus, RNA splicing deregulation represents an innovative and exciting area of research, in that it might be possible to modify or regulate RNA as novel anticancer strategy.

AS is controlled by spliceosome, which is a dynamic molecular machine consisting of small nuclear RNA (snRNA) and various protein complexes that cycle on and off from pre-mRNA during intronic splicing. This nuclear complex is composed of at least 170 proteins and five snRNA associated with proteins forming the U1, U2, U4, U5, and U6 small nuclear ribonucleoproteins (snRNP). It removes an intron from the primary transcript and subsequently joins the exons by a trans-esterification reaction; the intron then undergoes debranching and is subsequently degraded. ${ }^{8}$ The accuracy of this process is essential for normal cellular function, whereas alternative splicing deregulation often occurs in tumors. As a result, modulation of this tumor hallmark is now emerging as a promising strategy for anti-cancer therapies..$^{9,10}$ Changes in alternative splicing are frequently caused by point mutations in the splicing factors such as SF3B1, which occurs in a wide range of tumors, including chronic lymphocytic leukemia (CLL), myelodysplastic syndromes (MDS), melanoma and breast cancers, resulting in aberrant splicing. ${ }^{11-}$ ${ }^{13}$ Also, MM cells harbor somatic alterations in this driver gene with missense mutations (K700 and K666) observed in about $1.7 \%$ of patients. ${ }^{14}$ Importantly, despite low mutational burden, recent studies suggest RNA splicing deregulation as a driver mechanism for disease progression and drug-resistance occurrence as well. ${ }^{15,16}$ Here, we explored the impact of small molecule modulators of the spliceosome in perturbing tumor cells survival pathways and provide evidence for a novel combination strategy to treat MM.

\section{Methods}

For a more detailed description of the methods used, see the Online Supplementary Appendix.

\section{Reagents}

Meayamycin B and Sudemycin D6 (SD6) were kind gifts from Prof. Kazunori Koide (University of Pittsburgh) and Prof. Thomas Webb (SRI Biosciences, Dept. of Chemistry and Biochemistry UCSC), respectively. E7107 was kindly provided by H3 Biomedicine, Inc.

\section{Cell lines and primary tumor specimens}

Cell lines were obtained from the ATCC or sources indicated in the Online Supplementary Appendix.

\section{Intracellular BH3 profiling}

Intracellular $\mathrm{BH} 3(\mathrm{iBH} 3)$ profiling was performed as described in ${ }^{17}$. Briefly, MM cells were pelleted and suspended in MEB buffer with addition of each $\mathrm{BH} 3$ peptide treatment with $0.002 \% \mathrm{w} / \mathrm{v}$ digitonin (MS1, mBAD and HRKy peptides were used). Mitochondria in the permeabilized cells were exposed to peptides for 45 minutes (min) at $26^{\circ} \mathrm{C}$ before fixation with $4 \%$ formaldehyde at room temperature for $10 \mathrm{~min}$. After addition of $\mathrm{N} 2$ neutralizing buffer for $15 \mathrm{~min}$, cells were stained with Alexa Fluor $^{\circledR} 488$ Mouse anti-Cytochrome c (Clone 6H2.B4, BD Biosciences) 1:40 in 2\% Tween20, 10\% bovine serum albumin phosphate-bufferd saline for 2 hours at room temperature and then over night at $4^{\circ} \mathrm{C}$. The quantification of Cytochrome c loss induced by each peptide was evaluated by flow cytometry (Navios 10/3, Beckman Coulter). Values indicate the percentage of Cytochrome c-negative cells calculated as in ${ }^{17}$.

\section{Transcriptome profiling, alternative splicing and pathway analysis}

RNA samples from $\mathrm{H} 929$ cells treated or not with Meayamycin B ( $3 \mathrm{nM}, 8$ hours) were isolated in duplicate for each condition with the miRNeasy mini kit (Qiagen, \#1038703) and processed using WT PLUS Reagent Kit, according to the manifacturer's protocol (Thermo Fisher). Wide mRNA-transcriptome profiling was assessed using ClariomD Human array (Thermo Fisher). Robust Multi Array (RMA) normalization on raw data, transcript annotation (Clariom_D_Human.r1.na36.hg38.a1.transcript) and AS analysis were performed using Transcriptome Analysis Console (TAC 4.0) software (Thermo Fisher). AS events were identified in Meayamycin B-treated versus untreated H929 cells after filtering for transcripts expressed in both conditions, exon expressed in at least one condition, Exon Splicing Index of at least $2(<-2$ or $>2)$, exon $P$-value $<0.05$. Pathway enrichment analysis of significant alternative spliced genes was performed with the fgsea package in $\mathrm{R}$ to- 
gether with BIOCARTA and REACTOME datasets. Significant pathways were selected based on false discovery rate (FDR) q-value $<25 \%$. Global transcript expression levels were submitted on gene expression omnibus (GEO) data repository, under GEO accession number GSE167042.

\section{In vivo mouse models}

All in vivo experiments were performed in accordance with the laws and institutional guidelines for animal care, approved by the Institutional Animal Care and Use Committee of University Hospital San Martino (protocol \#473). Five-week-old female NOD/SCID J mice were acquired from Charles Rivers Laboratories (France) and were accli-

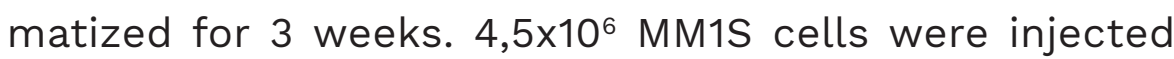
subcutaneously in both flanks of each mouse. Treatment was initiated when the tumors reached a volume of about $50 \mathrm{~mm}^{3}$. In each experiment, mice were randomly divided to one of the following groups: control (vehicle); Venetoclax $100 \mathrm{mg} / \mathrm{kg} /$ day, dissolved in vehicle $(60 \%$ phosal 50 PG, $30 \%$ polyethylene glycol $400,10 \%$ ethanol) and administered by oral gavage; SD6 $12 \mathrm{mg} / \mathrm{kg} /$ day, formulated in vehicle (10\% hydroxypropyl-beta-cyclodextrin [HPCD] in phosphate buffer $\mathrm{pH}$ 7.4) and intra-tumorally injected; E7107 2,5 mg/kg/day prepared in vehicle (10\% ethanol, $5 \%$ Tween-80, Quorum sensing with saline) and intravenously injected (i.v.). Schedules are described in the individual figure legends. Tumor volume was calculated using the formula: tumor volume $=(w 2 \times W) \times \pi / 6$, where " $w$ " and "W" are "minor side" and "major side" (in $\mathrm{mm}$ ), respectively. Mice were sacrificed when the tumor reached a volume of about $1,5 \mathrm{~cm}^{3}$. Tumor masses were always isolated at the end of the experiment, weighted and fixed in $10 \%$ neutral buffered formalin ( $v / v)$ for histology.

\section{Results}

\section{Splicing machinery is markedly deregulated in multiple myeloma and represents a disease aggressiveness biomarker}

Genome-wide studies have recently demonstrated that deregulated expression of genes involved in splicing acts as driver event for numerous tumors including MM. ${ }^{7,18}$ These data have prompted a growing interest for drugging the spliceosome machinery as a novel strategy to improve anti-cancer therapies. ${ }^{14-16}$ We explored transcription levels of core snRNP assembly genes in primary MM cells from publicly-available collections of gene expression datasets by applying the KEGG spliceosome signature..$^{14,19-21} \mathrm{~A}$ progressive transcriptional increase was observed in more advanced disease phases from monoclonal gammopathy of undetermined significance (MGUS) to plasma cell leukemia (PCL) and in human multiple myeloma cell line (HMCL) compared to normal plasma cells, indicating an association with tumor progression route (Online Supplementary Figures S1 and S2). We confirmed higher expression in MM cells by gene expression analysis in primary cells from seven additional MM patients and peripheral blood mononuclear cells (PBMC) from two healthy donors (Figure 1A). We next extended the analysis to the MMRF (MM Research Foundation) CoMMpass study that includes $774 \mathrm{MM}$ patients ${ }^{5,22}$ profiled by RNA sequencing by standardizing KEGG spliceosome signature with a zscore method, ${ }^{5}$ which stratified MM cases into three groups: low $(n=303)$, intermediate $(n=126)$ and high $(n=345)$ (Figure 1B). Then we sought to explore any potential impact of aberrant spliceosome signature on clinical outcome and found that, in line with reported data, ${ }^{16} \mathrm{pa}-$ tients in the high z-score group showed shorter progression-free survival (PFS) and overall survival (OS) when compared with low-z score patients (median PFS 1,300 days vs. 700 days, $P=0.0017$; median survival not-reached vs. 2,000 days, $P=0.00027$ ) (Figure $1 C$ ). Importantly, a univariate Cox-model analysis confirmed a spliceosome signature role in predicting OS $(P=0.0012)$ and $\mathrm{PFS}(P=0.0021)$, similarly to ISS stage III $(P=0.0001)$, 1q gain/amp $(P<0.0001)$, del13q/RB1 $(P=0.0002)$, and TP53 mutation occurrence $(P<0.0001)$ (Figure 1D; Online Supplementary Figure S3). Overall, our transcriptome analyses revealed a group of MM patients with higher upregulation in the RNA splicing machinery genes which correlated with poor prognosis. The spliceosome is a dynamic molecular machine consisting of several nuclear protein complexes, with the splicing factor SF3B1 responsible for on/off cycle of pre-mRNA during intronic splicing, essential for the correct functioning of this machinery. As result, western blot, immunohistochemistry and immunofluorescence analyses revealed high levels of SF3B1 on MM cell lines and CD138+ from patient-derived bone marrow biopsies (Figure 1E and F; Online Supplementary Figure S4) which was further increased upon interaction with bone marrow stromal cells (BMSC) (Online Supplementary Figure S5). Notably, the greater SF3B1 protein level observed in tumor cells could be explained, at least in part, by the higher proliferation rate (in term of $S$ and $G 2 / M$ phases residing cells) of MM compared with healthy donor counterpart cells. (Online Supplementary Figure S6). Altogether these data suggest a role for spliceosome deregulation in MM growth.

\section{Targeting the spliceosome core-element SF3B1 results in broad anti-multiple myeloma activity}

Comprehensive RNA sequencing-based analyses have revealed profound and significant transcriptome changes, including alternative pre-mRNA splicing between MM and normal samples, with significant impact on overall clinical outcome. ${ }^{14}$ Despite that, SF3B1 somatic mutations, resulting in loss of function, occur in only $1.7 \%$ of MM patients, thus 
suggesting additional events accounting for the alterations observed in MM cells. ${ }^{15}$ Therefore, we evaluated the spectrum of alternatively spliced events (ASE) in MMRF database, irrespective of SF3B1 mutation status. We classified the ASE into five categories and analyzed the frequency of pattern changes according to SF3B1 expression level (top vs. bottom quartile) (Figure 2A). Wide variability was observed, with alternative transcription start site (ATSS), intron retentions (IR) and alternative transcription termination site (ATTS) as the most abundant ASE among the SF3B1 highly expressed group, indicating widespread spliceosome deregulation in $\mathrm{MM}$, regardless of specific mutational profiling. Based on these data, we next investigated SF3B1 role in these cells by genetic perturbation in a panel of MM cell lines. According to the reported activity of this factor in binding and splicing pre-mRNA, ${ }^{9}$ immunofluorescence analysis for the SR (serine/arginine-rich) protein $\mathrm{SC}-35$ revealed a significant modulation of splicing machinery after SF3B1 knockdown $(K D)$, with KD cells exhibiting reduced number of speckles which resulted larger and darker than control (Figure 2B). Importantly, SF3B1 KD not only affected splicing but also impaired MM cell viability by increasing apoptotic cell death in different MM cell lines and inducing caspase-3 and PARP1 cleavage as well (Figure 2C; Online Supplementary Figure S7). We subsequently tested the activity of the splicing modulator Meayamycin B in a panel of 14 MM cell lines,
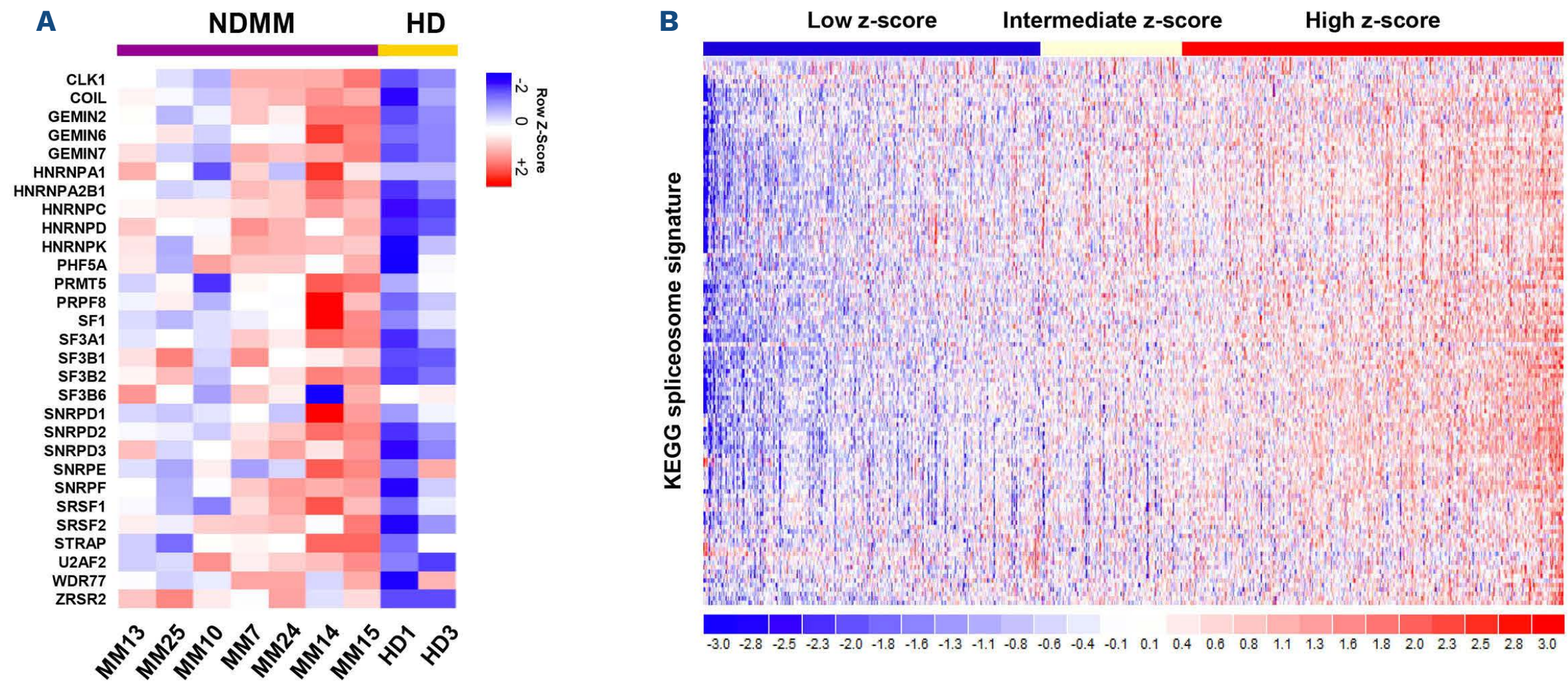

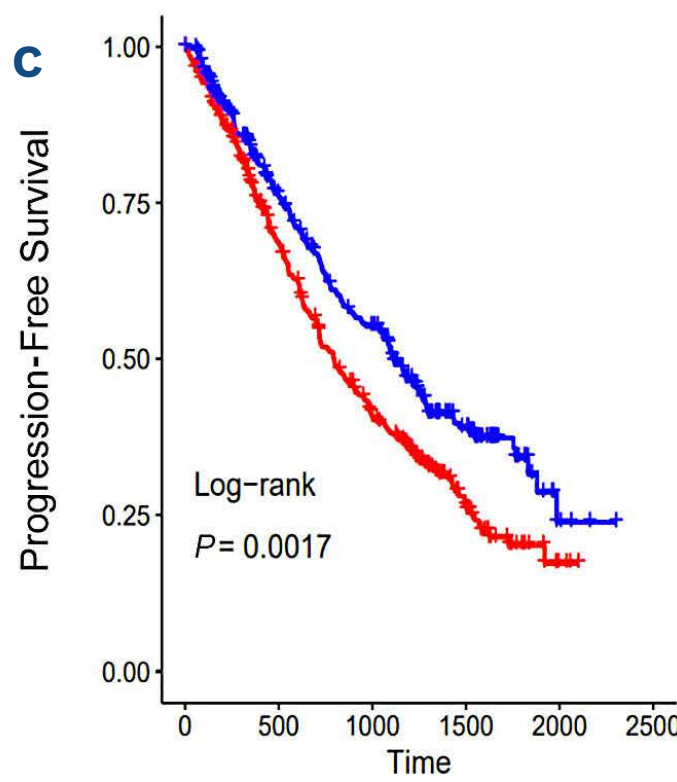

Number at risk

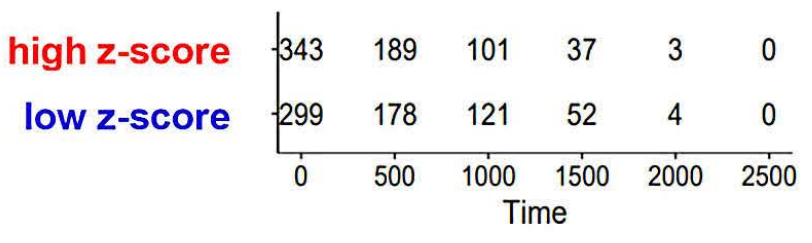

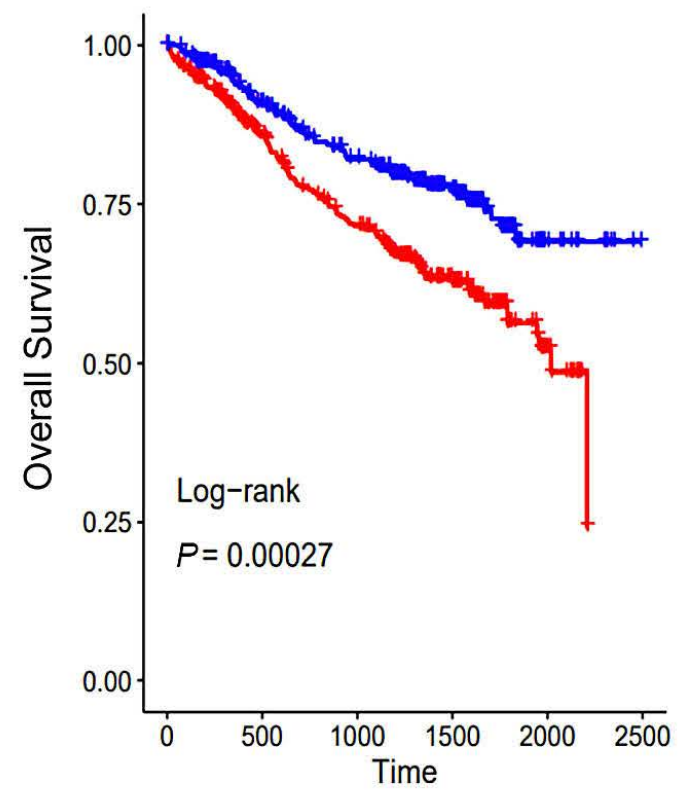

Number at risk

\begin{tabular}{|c|c|c|c|c|c|}
\hline 343 & 240 & 188 & 105 & 16 & 0 \\
\hline 299 & 217 & 175 & 107 & 14 & 0 \\
\hline 0 & 500 & & 1500 & 2000 & \\
\hline
\end{tabular}

Continued on following page. 


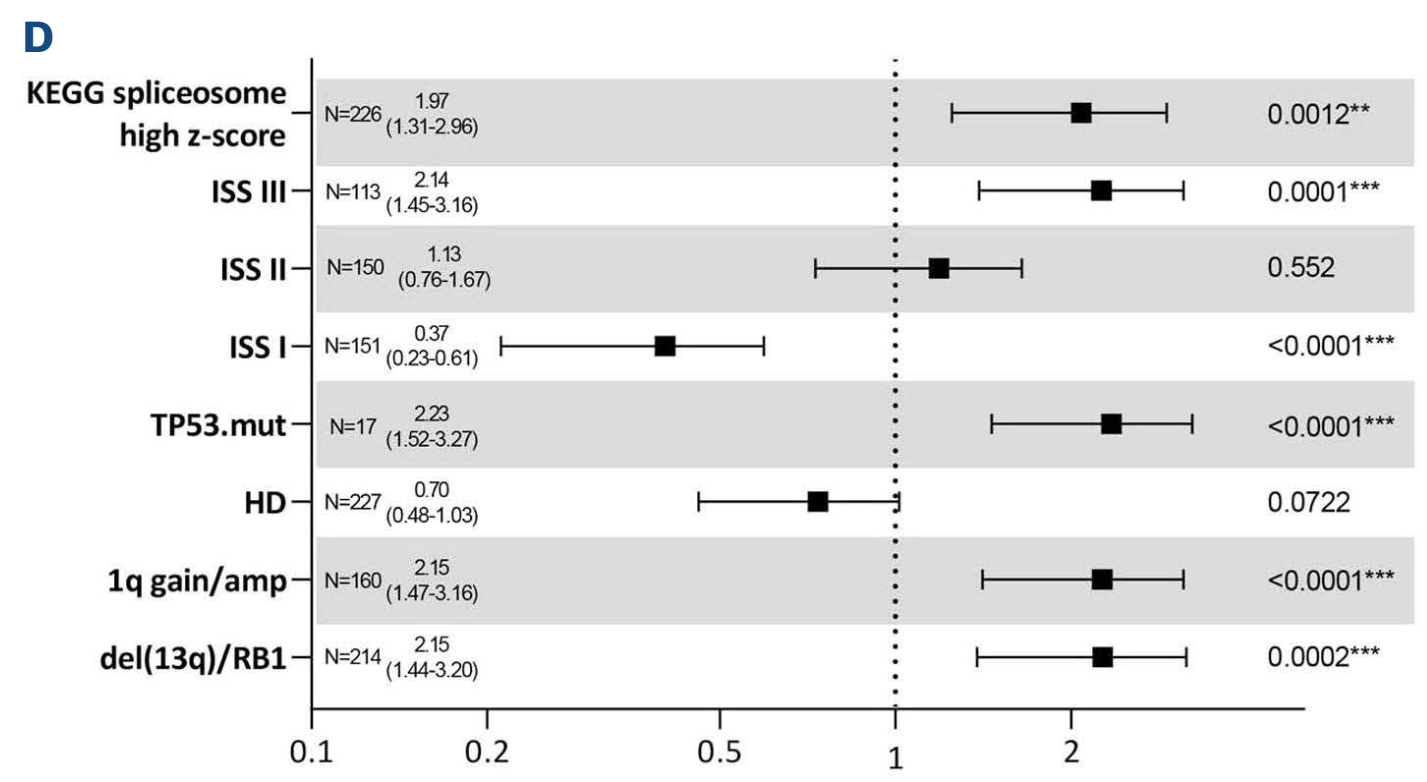

$\mathbf{E}$
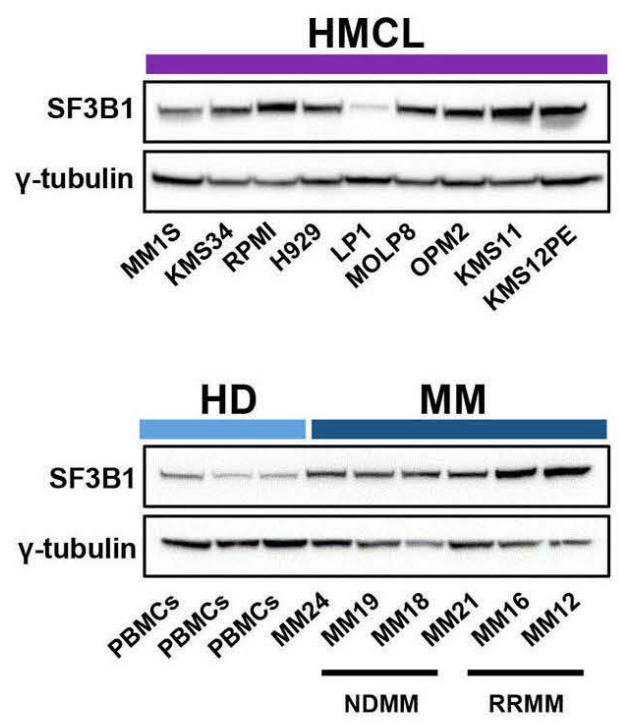

$\mathbf{F}$ MM1

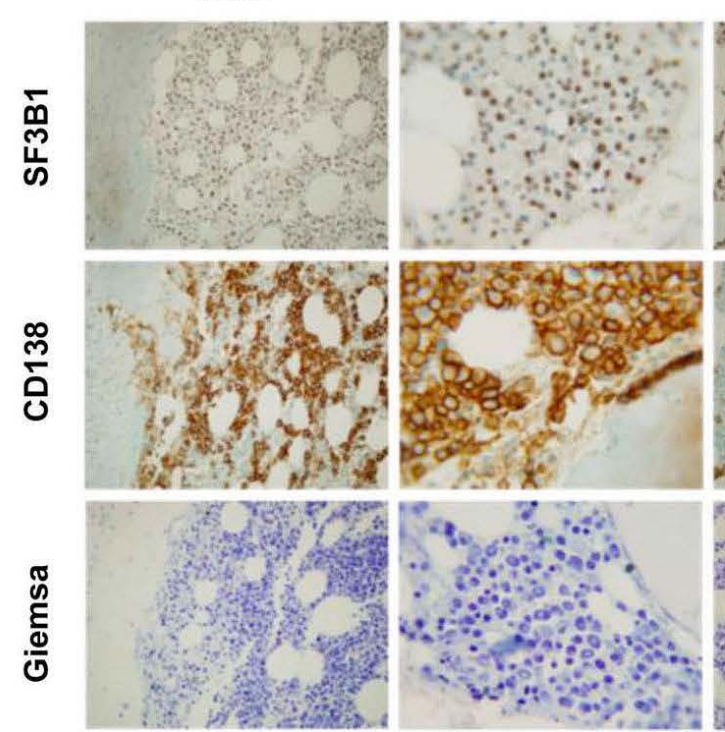

MM3

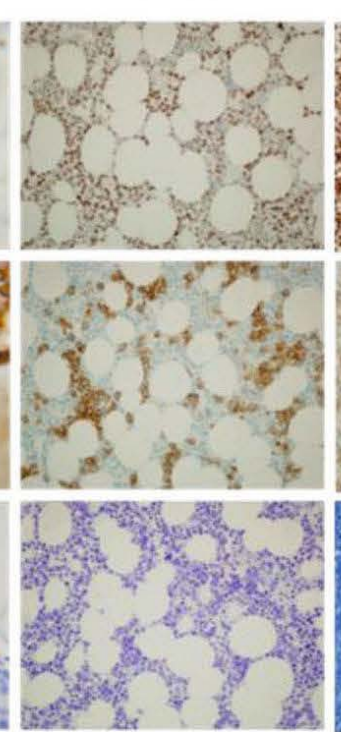

MM4

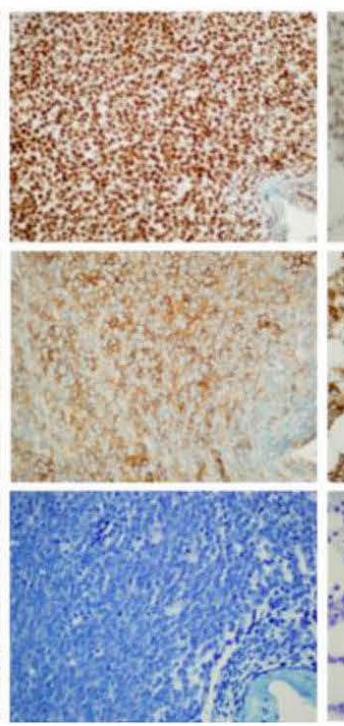

MM5

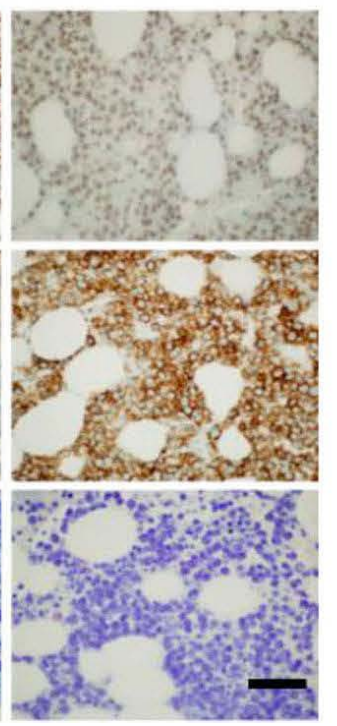

Figure 1. Splicing machinery is markedly deregulated in multiple myeloma. A) Heat map showing expression levels (row z-score) of indicated genes among plasma cells derived from patients with multiple myeloma $(M M)(n=7)$ and peripheral blood mononuclear cells (PBMC) from healthy individuals $(n=2)$. The color scale spans the relative gene expression changes standardized on the variance. B) Heatmap of $774 \mathrm{MM}$ patients included in CoMMpass study ordered in 3 groups according to z-score calculated on 124 genes of KEGG spliceosome gene set, as annotated in MSigDB. C) Kaplan-Meier survival curves of high z-score (red) and low z-score (blue) MM patients of CoMMpass cohort (642 patients analyzed) on progression-free survival (PFS) and overall survival (OS) data. Log-rank test $P$-value and number of samples at risk in each group across time are reported. D) Forest plot based on Cox univariate analysis for OS. Squares represent hazard ratios; bars represent $95 \%$ confidence intervals. E) Protein lysates from a panel of MM cell lines (human multiple myeloma cell line [HMCL]) and primary MM patients or healthy donors (HD) were analyzed for SF3B1 expression by western blotting. GAPDH was used as loading control. One representative experiment is shown. F) Representative images of SF3B1 and CD138 immunocytochemistry stain in bone marrow (BM) from MM patients ( $\mathrm{n}=5$ ). Conventional Giemsa staining is also shown. Original magnification $\times 200$, scale bar, $100 \mu \mathrm{m}$.

where we observed a significant decrease of cell viability with half maximal inhibitory concentration $\left(\mathrm{IC}_{50}\right)$ values ranging from to 0.49 to $3.5 \mathrm{nM}$ (Figure $2 \mathrm{D}$ and E). Similar results were observed with additional splicing modulators such as Sudemycin D6 and E7107 (Online Supplementary Table S1). ${ }^{23-}$ ${ }^{25}$ The effect of Meayamycin B was next tested in primary MM cells, cultured in the absence or presence of the bone marrow microenvironment. As seen in Figure $2 \mathrm{~F}$ and $\mathrm{G}$ and the Online Supplementary Figure S8, primary MM cells were significantly depleted after treatment with Meayamycin B, while a minimal toxicity was observed on normal components of the bone marrow milieu. These data support SF3B1 as a promising therapeutic target in MM.

\section{Spliceosome deregulation affects genome stability of multiple myeloma cells, irrespective of Myc status}

Genomic instability is a tumor hallmark resulting from deregulated DNA damage response, DNA repair defects, and failure of cell-cycle checkpoints. Recent studies have revealed that alternative splicing reprogramming after DNA damage responses relies on regulation of RNA-binding proteins (RBP), which directly bind specific pre-mRNA and mRNA sequences and act as gatekeepers of genomic integrity. In this context, post-transcriptional RNA processing may add another layer of complexity to the maintenance of genomic stability in MM cells and targeting spliceosome during DNA damage response may rep- 
resent an innovative approach to sensitize tumor cells to genotoxic agents. ${ }^{26}$ As shown in Figure $3 A$ and $B$, we observed that in SF3B1 KD MM cells increased DNA doublestrand breaks and resulted in defective repair mechanisms as shown by accumulation of $\mathrm{\gamma H} 2 \mathrm{AX}$ and reduced RAD51 levels, respectively. These changes were similarly observed after drug-treatment with Meayamycin $B$ in a dose- and time-dependent manner (Figure 3C and D) and persist also after pre-incubation with pan-caspase inhibitor zVAD-fmk, thus suggesting a negligible role of apoptosis activation in the genomic instability triggered by spliceosome modulators (data not shown). Based on these data, we tested sensitivity of KD-SF3B1 MM cells to melphalan-induced DNA damage and, in line with previous studies, ${ }^{26}$ we found that drug exposure led to increased $\gamma \mathrm{H} 2 \mathrm{AX}$ induction, also in SF3B1-depleted cells, (data not shown) suggesting that huge spliceosome de- regulation, triggered by SF3B1 targeting, accounts for the enhanced anti-MM activity of genotoxic stress. Recent genome-wide MYC-synthetic lethal screens have identified spliceosome components as candidate genes for synthetic lethality strategies, suggesting these as exploitable vulnerabilities for MYC-driven cancers. ${ }^{27}$ In order to determine whether MYC presence correlates with Meayamycin $B$ efficacy, we compared its protein level with the specific $\mathrm{IC}^{50}$ value measured for each tested cell line. As shown in Figure 3E no significant correlation ( $R 2=0.1261, P=0.348$ ) was observed thus suggesting that Meayamycin $B$ acts regardless of MYC protein level. Similarly, the ectopic expression of MYC in U266 MM cell lines increased $\gamma-\mathrm{H} 2 \mathrm{AX}$ levels (Figure 3F) but slightly enhanced the anti-MM activity of chemical or genetic SF3B1 targeting (Figure 3G and $\mathrm{H}$ ), thus confirming the marginal role played by this oncogenic program on anti-MM activity of splicing modulators.
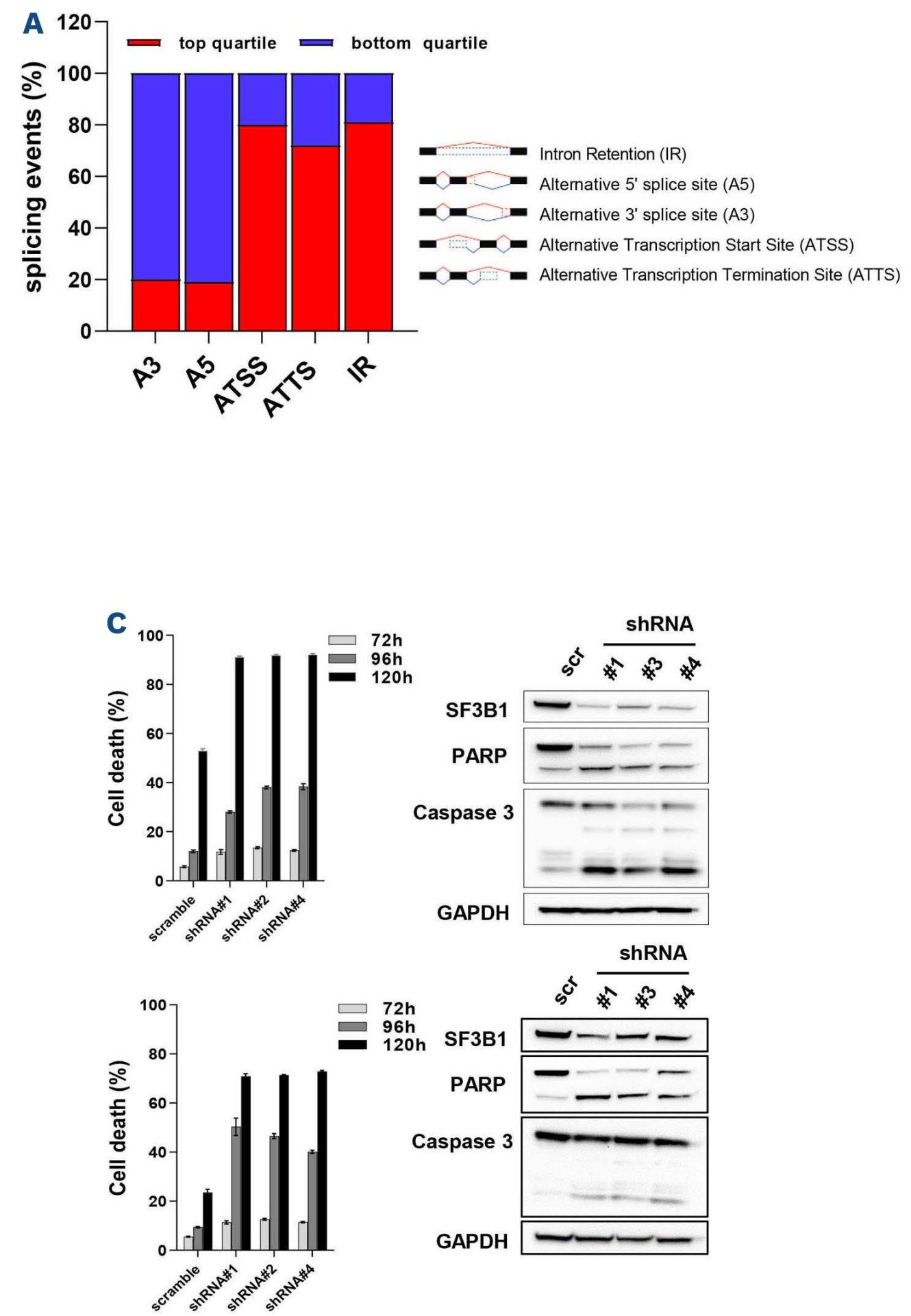
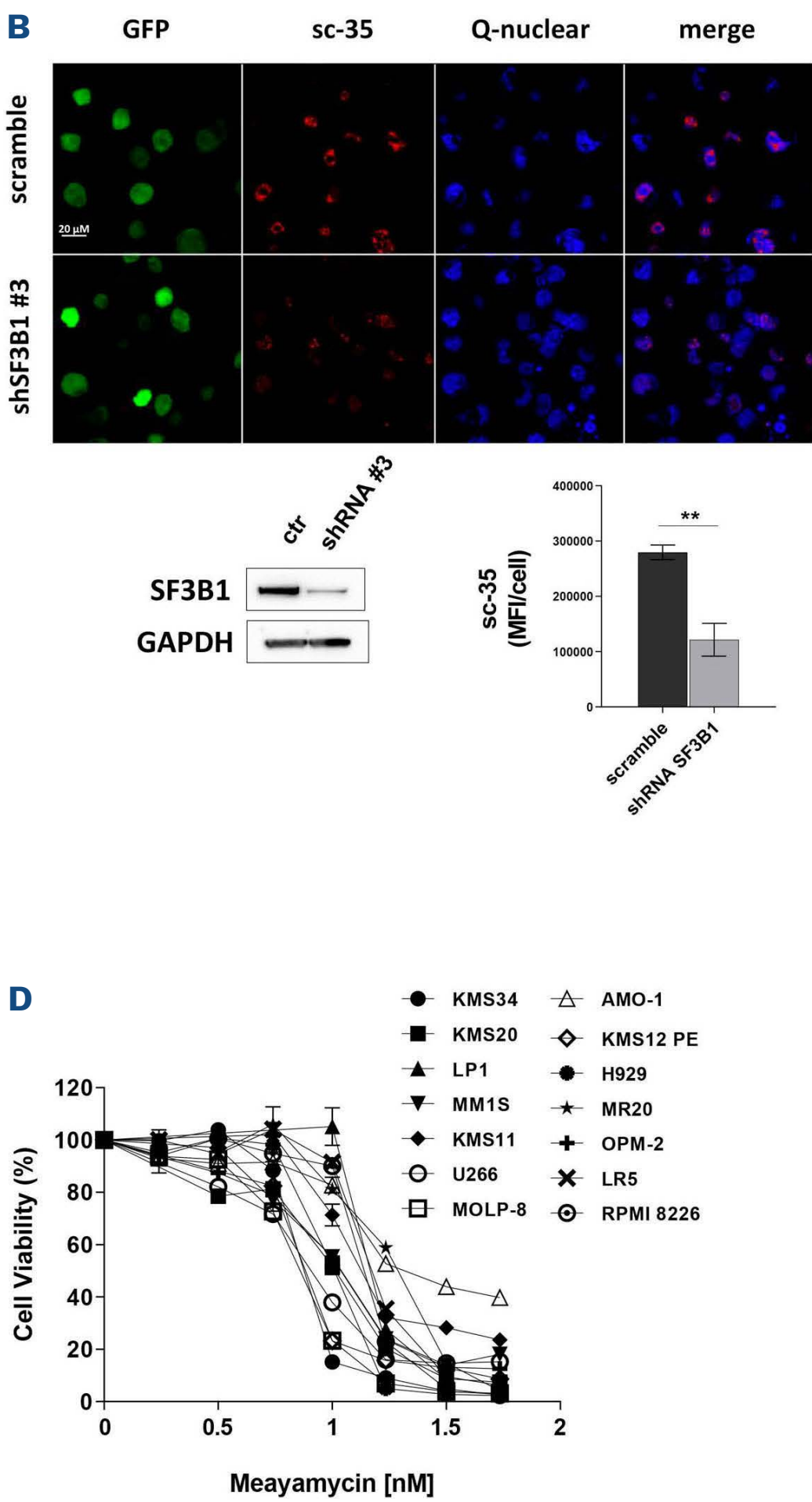

Figure 2. Continued on following page. 
E

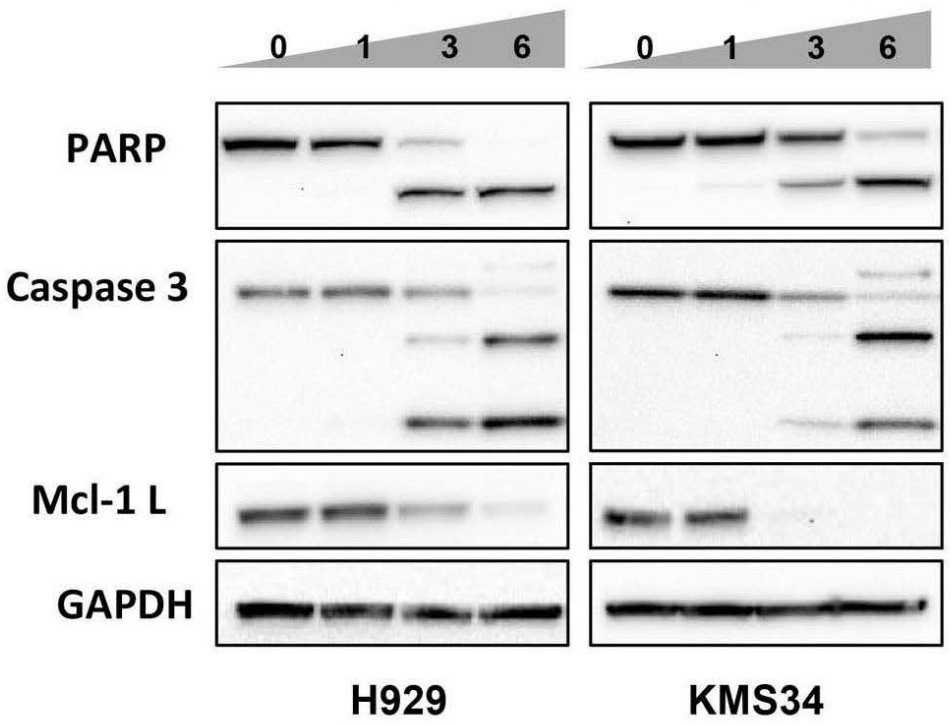

$\mathbf{F}$

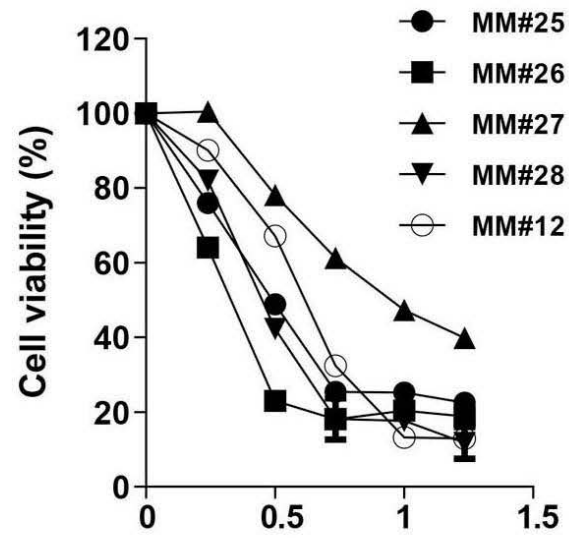

Meaymicin B, Log [nM]

G

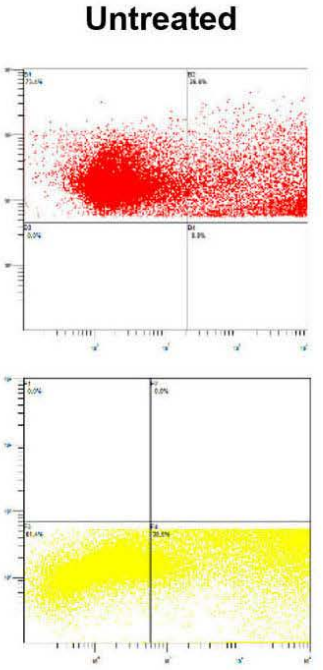

AV FITC
Meayamycin B [3nM]

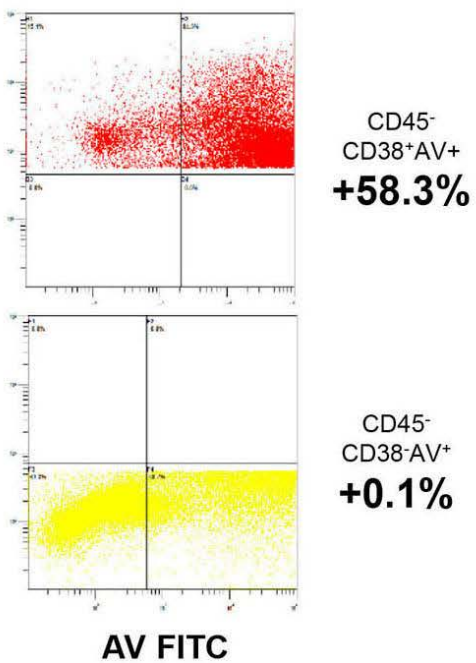

Figure 2. Spliceosome core-element SF3B1 targeting results in anti-multiple myeloma activity. A) Percentage of indicated splicing categories across RNA sequencing data derived from CoMMpass study according to SF3B1 expression levels (top vs. bottom quartile). B) Immunofluorescence staining for SC-35 in scramble and short hairpin (sh) SF3B1-sh RNA H929 multiple myeloma (MM) cells. SF3B1 silencing was validated by western blot as shown in the panel below. SC-35 staining of nuclear speckles is shown: mean fluorescence intensity (MFI) per cell/nucleus of the specific signal was quantified by counting at least 50 nuclei per condition as reported in the histogram below ( $* * P=0.0042$, two-sided Student $t$-test). Scale bar, $10 \mu \mathrm{m}$. C) MTS assay of MM1S (top) and H929 (bottom) lentivirally transduced with shSF3B1 (\#1,\#3 and \#4) or sh scramble. Cell viability was measured at indicated time point after transduction. Western blot analyses were performed at day 3 , confirming decreased SF3B1 protein levels and apoptotic cell death features (PARP1 and caspase 3 cleavage). Data are representative of at least 3 independent experiments. D) Cell viability curves compare a panel of $14 \mathrm{MM}$ cell lines' sensitivity to Meaymicin B (nM) for $48 \mathrm{~h}$ $(n=3$ technical replicates; mean $+/-$ standard deviation [SD]) E) Evaluation of PARP, caspase 3, MCL1 and GAPDH by western blot on indicated MM cell lines treated with increasing doses of MeaymicinB for 24 hours. F) Treatment of primary bone marrow aspirate samples from MM patients $(n=5)$ at various doses of MeaymicinB (0.1-30 nM) for 48 hours shows significant cytotoxicity of CD138+ tumor cells ( $n=3$ technical replicates; mean +/- SD). G) Ex vivo evaluation of Meaymicin $B$ in total bone marrow cells from one representative MM patient. After red cell lysis, cells were stained with Annexin V, DAPI and CD38 monoclonal antibody to identify viable as well as apoptotic myeloma (CD38 positive/CD45 negative) and normal (CD38 negative/CD45 negative) cells.

\section{Splicing inhibition remodels mitochondrial apoptotic dependencies in multiple myeloma cells}

In order to shed light on the molecular mechanisms mediating the effects of splicing modulators on MM cell growth, we evaluated the transcriptomic profiles in $M M$ cells treated with Meayamycin B. As shown in Figure 4A, we observed increased AS events compared to untreated cells, with intron retention (IR) and cassette exon (CE) as primary events across multiple targets $(41.97 \%$ and
$28.26 \%$, respectively). Splicing outliers including alternative 5' and 3' donor site as well as complex events were observed less frequently. A comprehensive enrichment analysis of BioCarta annotated pathways was employed to identify cellular pathways affected by this treatment. Significant enrichment was observed among biological processes involved in regulation of cell-cycle, disease progression, cell division and DNA replication, with apoptosis-related pathways identified as top-ranked events 
(Figure 4B). A gene level investigation, based on exon splicing index, revealed eleven genes of apoptosis pathway significantly mis-spliced: CHK1, BAX and BIRC3 were found to be interested by intron retention events, CASP10, CASP8 and CASP3 as affected by alternative cassette exons inclusion, PARP1 and MCL1 among targets with complex events and alternative 5' donor site, respectively (Figure 4C). Moreover, we confirmed a significant change in MCL1 transcripts with depletion of the anti-apoptotic long isoform (MCL-1L), and accumulation of pro-apoptotic short isoform (MCL-1s) following exposure to both Meaymicin B or Sudemycin D6 (Online Supplementary Figure S9). Other BCL2 family genes, including
BCL2 and BCLxL, were not affected (Figure 4D). A similar MCL-1 exon 2-skipped transcript occurred in SF3B1 silenced cells, with no modifications on BCL2 and BCLXL as well (Figure 4E); these changes were also confirmed at protein level (Figure 4F). Based on these data, we performed the intracellular $\mathrm{BH} 3$ (iBH3) profiling to investigate the mitochondrial apoptotic dependencies triggered by Meayamycin B or Sudemycin D6. ${ }^{28}$ As shown in Figure 4G both inhibitors resulted in significant increase of Cytochrome $c$ release following exposure to mBAD and HRKy $\mathrm{BH} 3$ peptides, indicating a functional shift in mitochondrial dependencies from MCL1 towards BLC2/BCLxL anti apoptotic members.
A

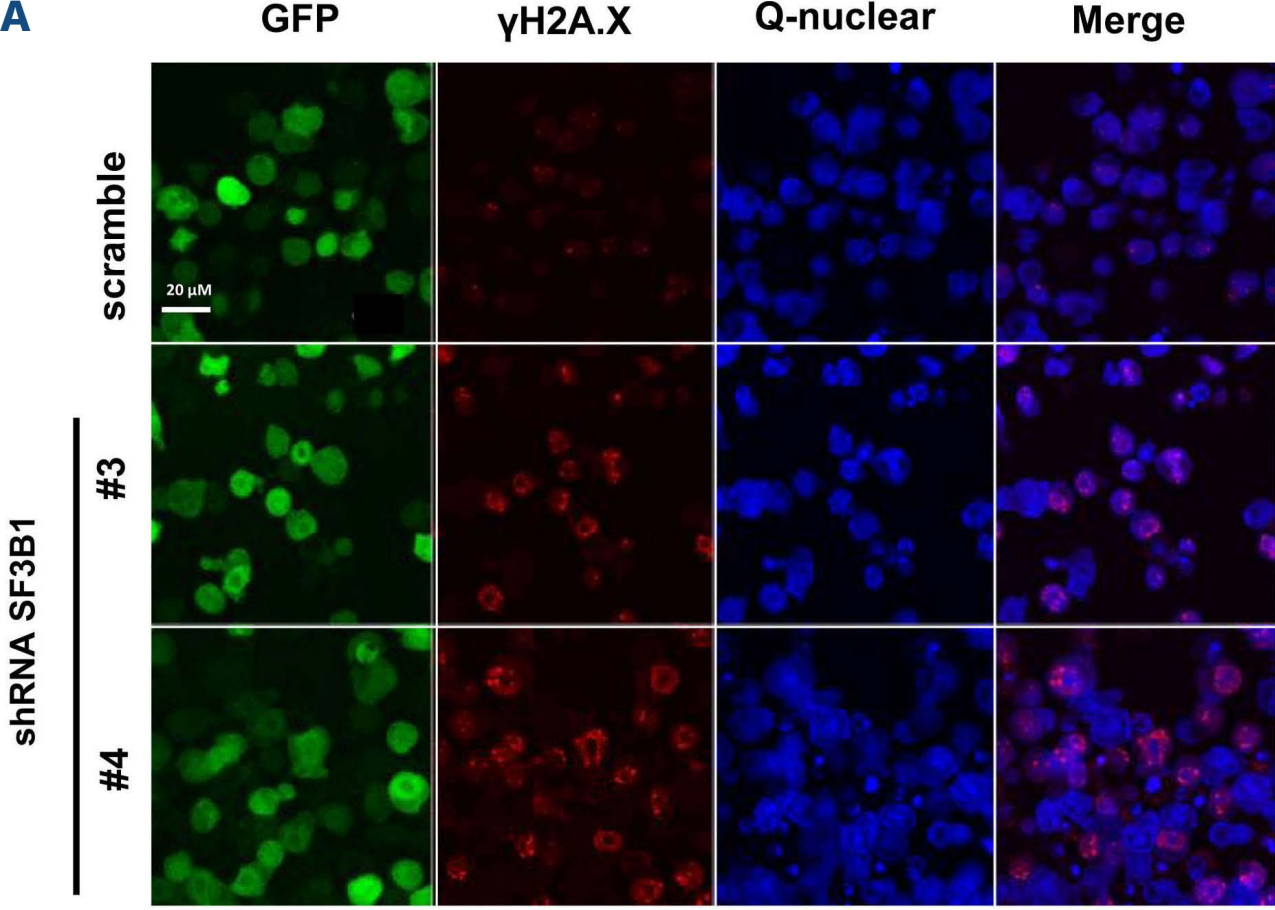

C

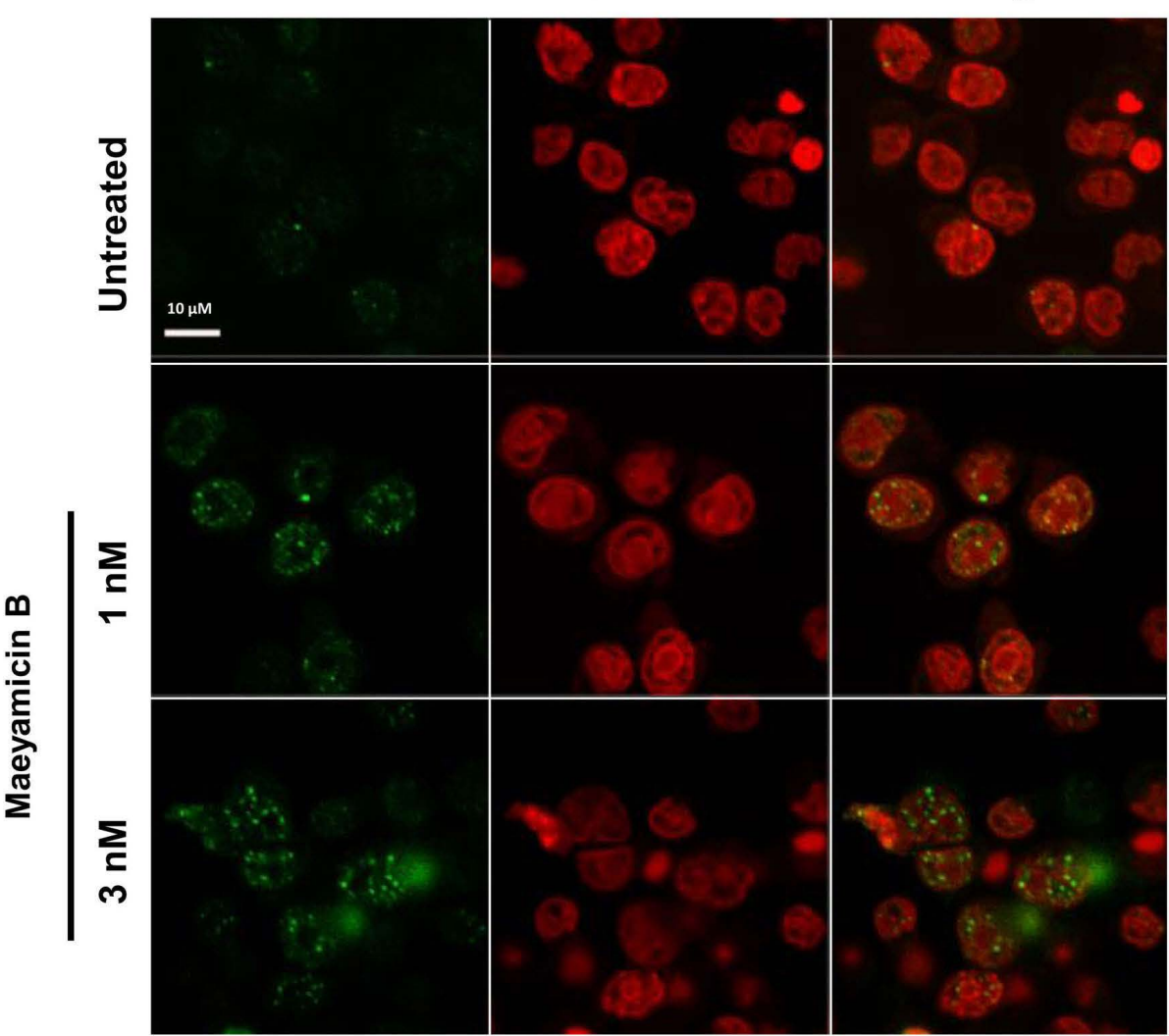

B

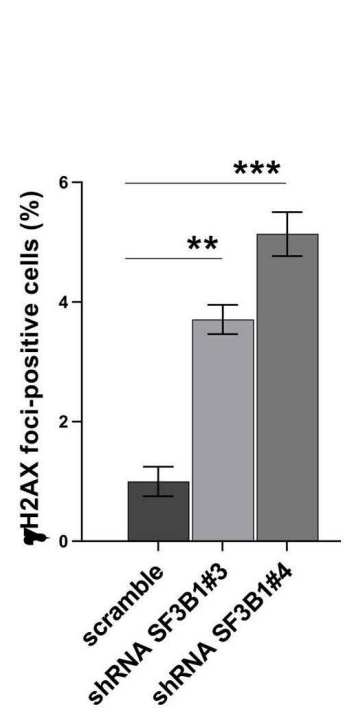

D

Meayamycin B (nM)

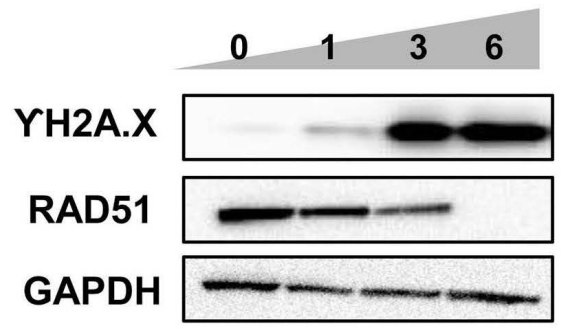

Time exposure (h)

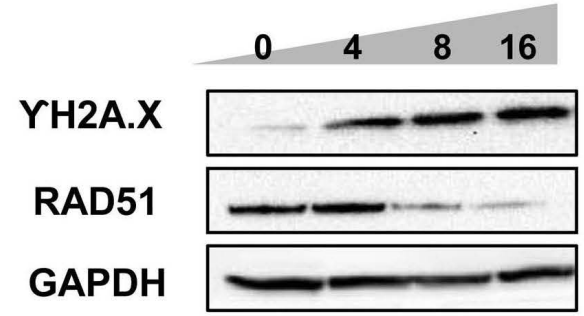



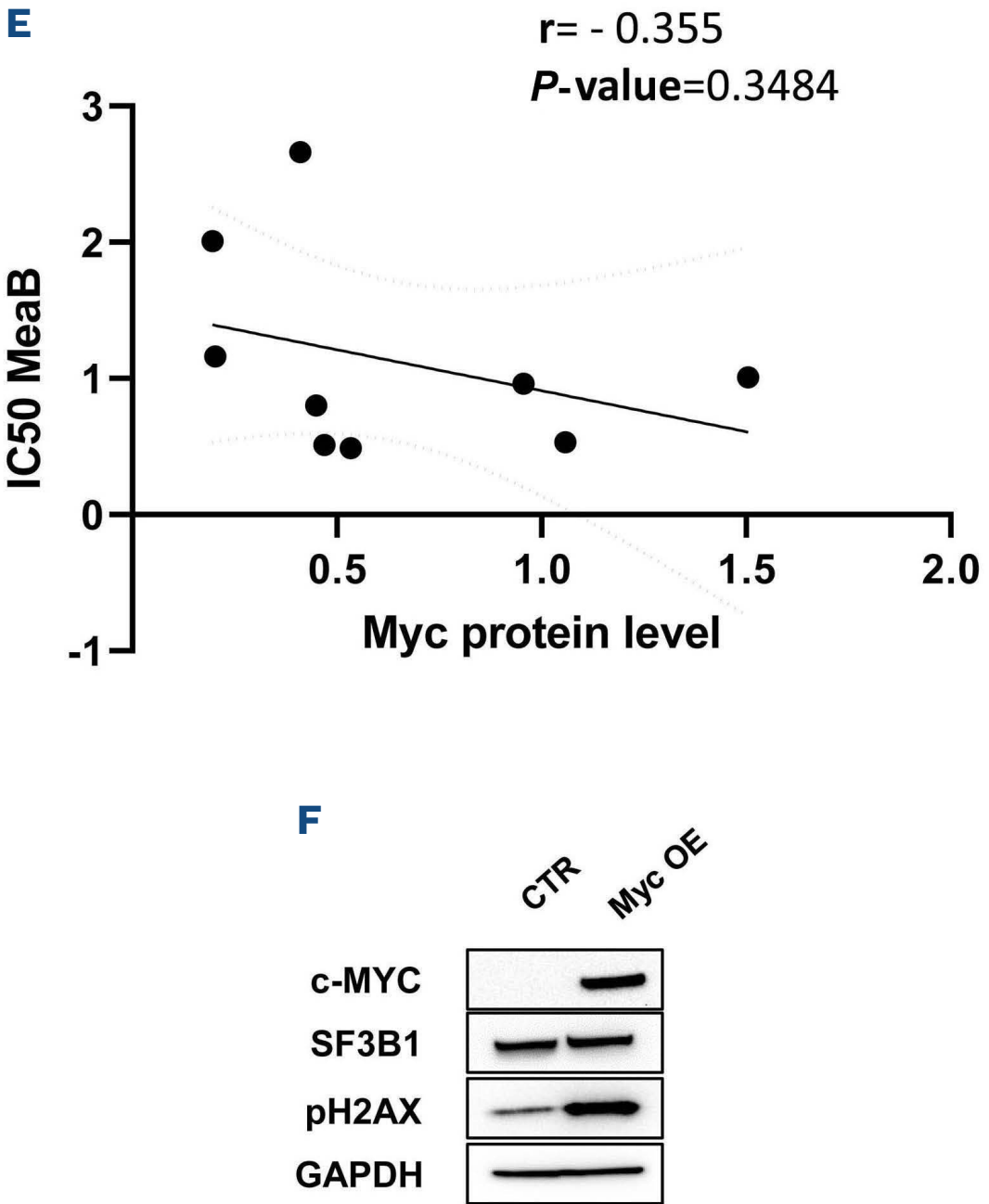

G
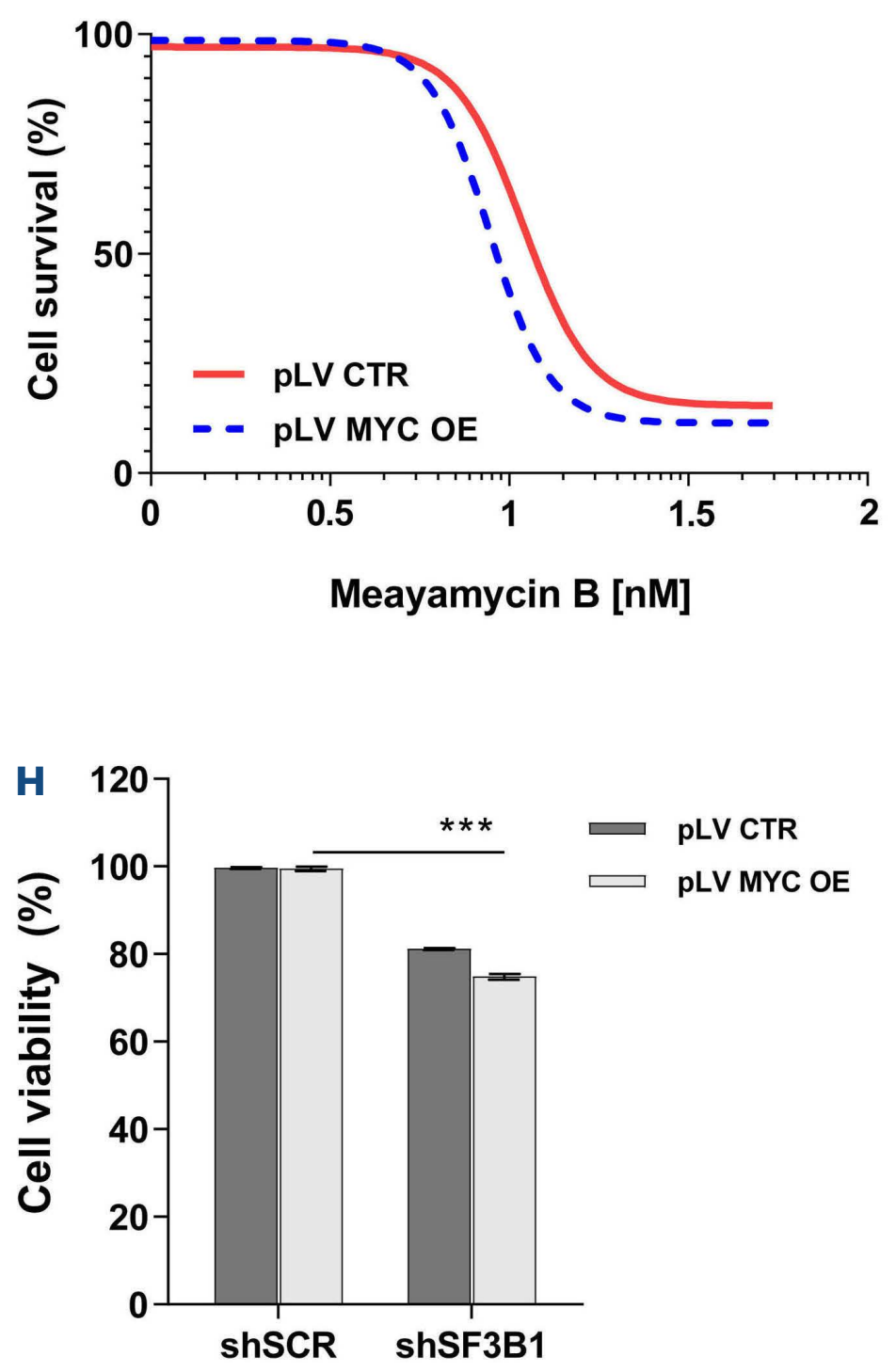

Figure 3. Spliceosome deregulation affects genome stability of multiple myeloma cells irrespective of Myc status. A) Detection of $\gamma \mathrm{H} 2 \mathrm{AX}$ and Q-nuclear was measured by confocal microscopy in H929 cells expressing short hairpin RNA (shRNA) (clone \#3 and \#4) targeting SF3B1 or control. Each panel includes representative foci-containing cells graph, over 3 experiments. ( $* * P=0.004$, $\star * \star P<0.001$; two-sided Student $t$-test). B) H929 cells were engineered to express an anti-SF3B1 shRNA (3 clones). Next, SF3B1, RAD51 and $\gamma \mathrm{H} 2 \mathrm{AX}$ protein levels were detected by immunoblotting. C) Detection of $\gamma \mathrm{H} 2 \mathrm{AX}$ and Q-nuclear by confocal microscopy of H929 cells ex-cultured with or without increased dosed of Meayamycin B (1-3 $\mu \mathrm{M})$ for 24 hours. Each panel includes representative foci-containing cells graph, over 3 experiments ( ${ }^{*} P=0.01, * \star \star \star * P<0.0001$; two-sided Student $t$-test). (A and $C$ ) scale bar, $50 \mu \mathrm{m}$. D) Western blot analysis of DNA damage response markers (RAD51 and $\gamma \mathrm{H} 2 \mathrm{AX}$ ) after Meaymicin B treatment over a range of doses (upper panel) and timing (3nM) (lower panel) in H929 cells. E) Relative expression of Myc protein plotted vs. Meaymicin $B$ cytotoxicity half maximal inhibitory concentration $\left(\mathrm{IC}_{50}\right)$ values. The Pearson correlation coefficient $(r)$ and the $P$-value, calculated using GraphPad Prism Version 5 analysis software, are indicated; F) immunoblot for cMyc, SF3B1 and $\gamma \mathrm{H} 2 \mathrm{AX}$ protein levels in isogenic U266 cells (pLV empty) or cMyc overexpressing (pLV cMyc) cells; G) these cells were treated with growing doses of Meayamycin B for 48 hours. Cell viability was measured with MTS assay and presented as a percentage of control. H) Cell viability analysis of pLV empty (pLV cMyc) U266 cells transduced with short hairpin RNA (shRNA) clones containing the target sequence of SF3B1 (clone\#1) or scrambled control. ( $* * P=0.004$; two-sided Student $t$-test).

\section{Splicing modulations increases sensitivity of multiple myeloma cells to the BCL2 inhibitor Venetoclax}

Cancer cells are dependent on multiple anti-apoptotic proteins to promote their survival, therefore targeting more than one protein results in synergistic effects in several preclinical models of solid and hematological tumors. ${ }^{29-32}$ In such a scenario, the dysregulated expression of BCL2 family members observed after splicing modulator treatment, prompted us to harness apoptosis as a strategy for enhancing anti-MM activity of these agents. We therefore evaluated the ability of splicing modulators to enhance effects of the BCL2 specific inhibitor Venetoclax against MM cell growth and survival. Low dose of this
BH3-mimetic and Meayamycin B or Sudemycin D6 combo was synergistic in several MM cells, including those carrying $\mathrm{t}(11 ; 14)$, which has been reported to affect anti-MM activity of Venetoclax. (Figure $5 \mathrm{~A}$ and $\mathrm{B}$; Online Supplementary Figure S10-11). ${ }^{33}$ As result, PARP1 and caspase 3 cleavage occurred after these stimuli at much extent compared with single agent exposure (Figure 5C). These results were also confirmed in SF3B1 KD MM cells (Figure 5D). We next examined the effects of the combination therapy on primary plasma cells (PC) collected from MM patients: as shown in Figure 5E we confirmed the increased sensitivity to Venetoclax of MM PC exposed to SD6 compared with single agent treatment, regardless of 
specific stage-disease, cytogenetic abnormalities, or previous drug exposure. Importantly, no significant effects were observed on PBMC derived from the same MM patients, suggesting tumor specificity of drugs combination. The impact of this strategy was finally tested on primary MM cells in the presence of their bone marrow microenvironment with slight toxicity observed on normal bone marrow components (Figure 5F; Online Supplementary Figure S12). In order to strengthen clinical relevance of our findings, we next screened different agents currently used for MM treatment in combination with splicing modulators, by performing head-to-head comparison analysis. As shown in the Online Supplementary Figure S13, Venetoclax was readily identified as the best sensitizer of MM cells to Meaymicin B activity with a Combination Index resulting to be the lowest among tested drugs. Collectively, these data support splicing modulation as a strategy to increase Venetoclax sensitivity of MM cells. Based on these results, we next focused on drug resistance mechanisms by investigating whether splicing pathways affect Venetoclax resistance of MM cells. To this aim, we assessed a panel of $10 \mathrm{MM}$ cell lines and measured absolute $\mathrm{IC}_{50}$ to capture the efficacy and potency of the drug (Figure $5 \mathrm{G}$ ). We then listed all cell lines according to Venetoclax sensitivity and used CCLE RNA sequencing data to compare gene expression profiling of resistant versus sensitive cell lines; significant de-regulated genes were submitted to the GSEA software to find most affected pathways. Importantly, this analysis revealed that gene sets associated with RNA biology, RNA processing, and splicing pathways, were strongly enriched in sensitive cell lines, based on negative normalized enriched score (NES) and low qvalue, thus supporting their link with venetoclax-sensitivity (Figure $5 \mathrm{H}$ ).

\section{Splicing inhibitors synergize with Venetoclax in vivo in multiple myeloma xenograft murine models}

We finally evaluated the efficacy of the combination regimen in vivo in MM xenograft murine models. In the first model, mice were treated with Venetoclax $(100 \mathrm{mg} / \mathrm{kg}$, oral administration, once a day for 15 days) and SD6 (12 $\mathrm{mg} / \mathrm{kg}$, intratumorally, once a day for 10 days) alone or in combination. SD 6 dosing was chosen based on previously reported efficacy and safety profiles in xenograft lymphoma models, while Venetoclax was used at $100 \mathrm{mg} / \mathrm{kg}$ to reduce its potency as single agent. Treatment was well

\section{A}

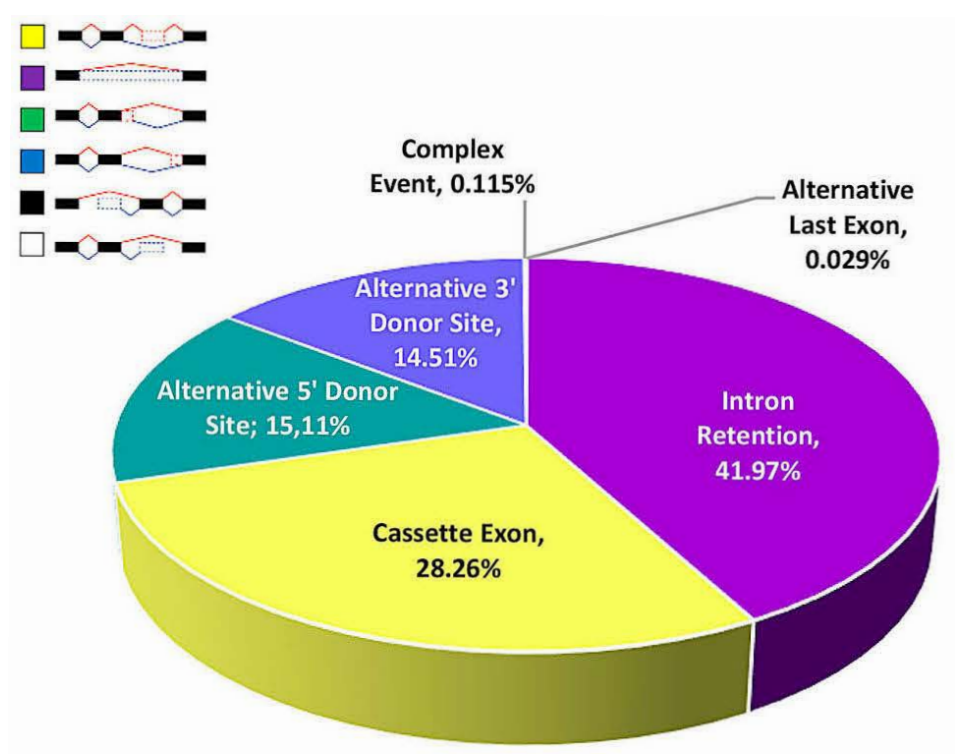

C

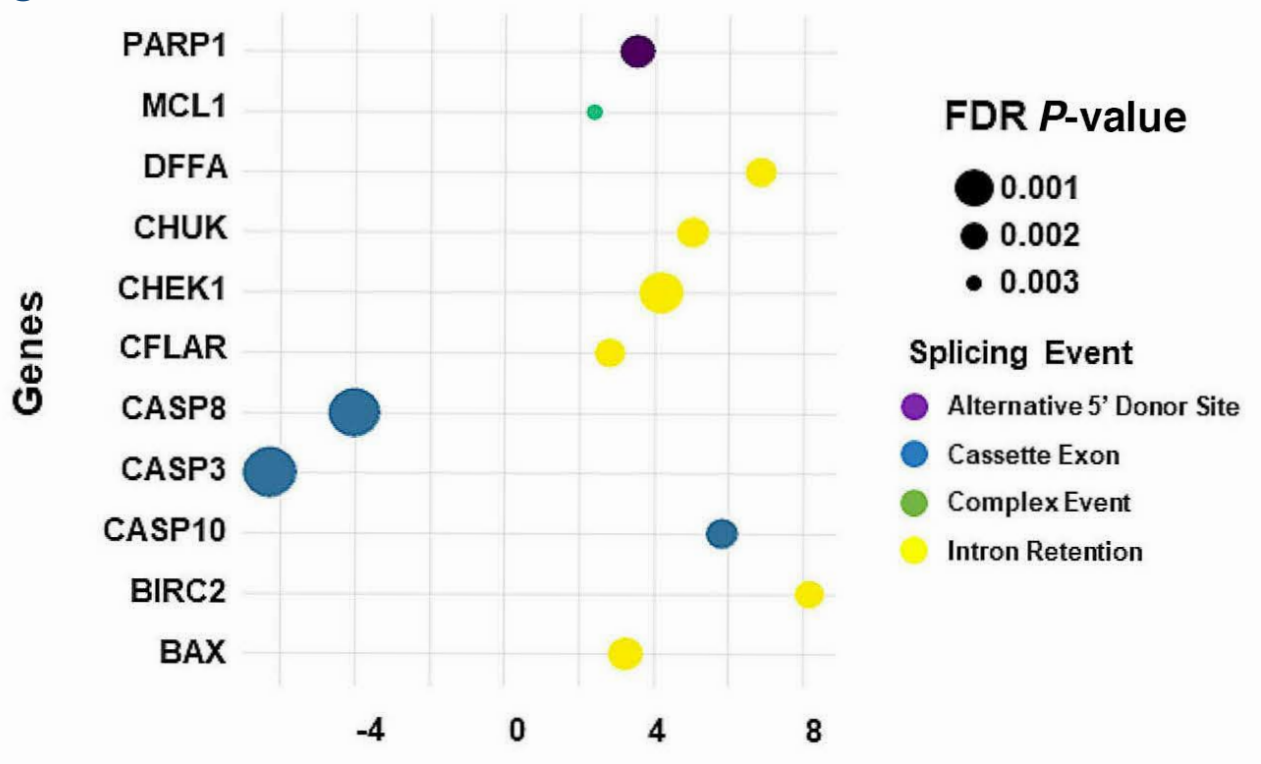

Exon Splicing Index
B

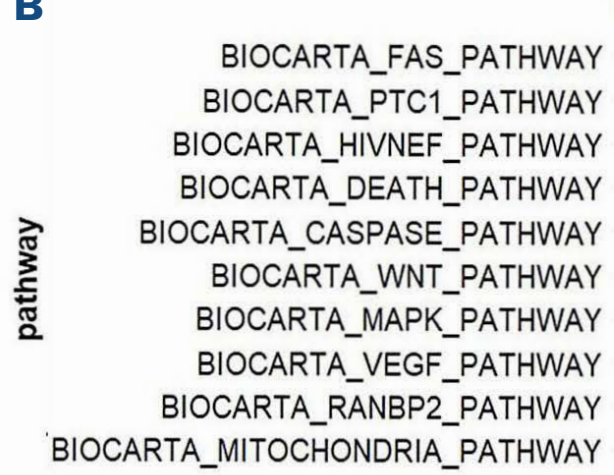

Enriched biocarta patwhays in significant spliced genes

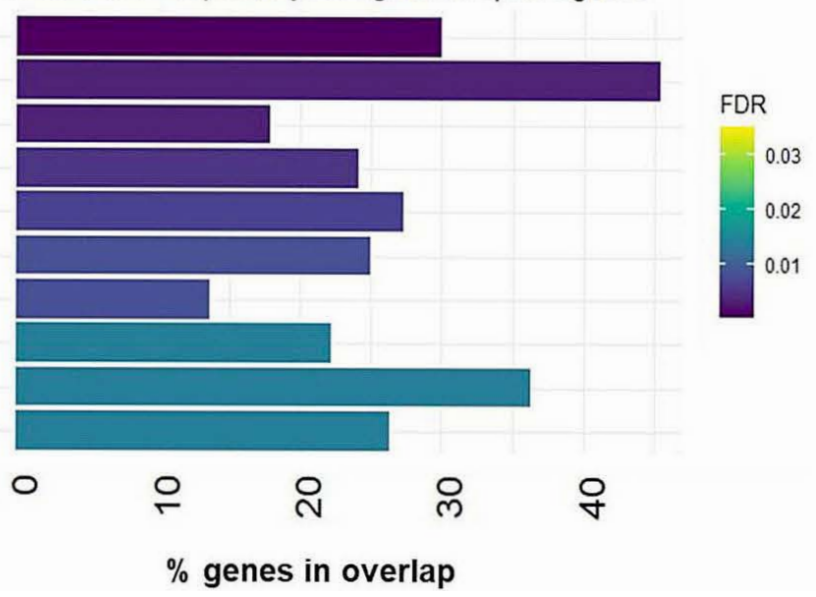

Figure 4. Continued on following page. 

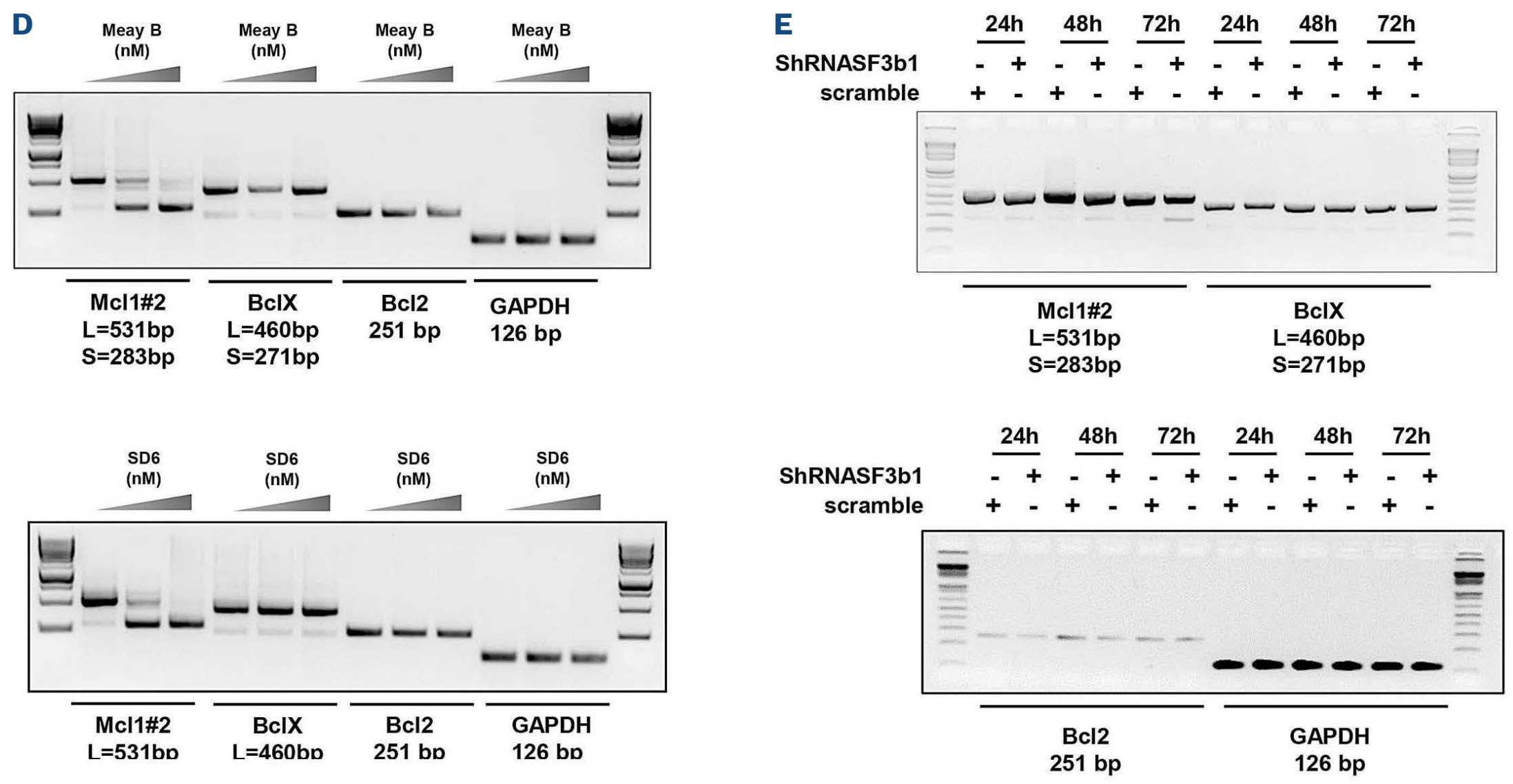

F

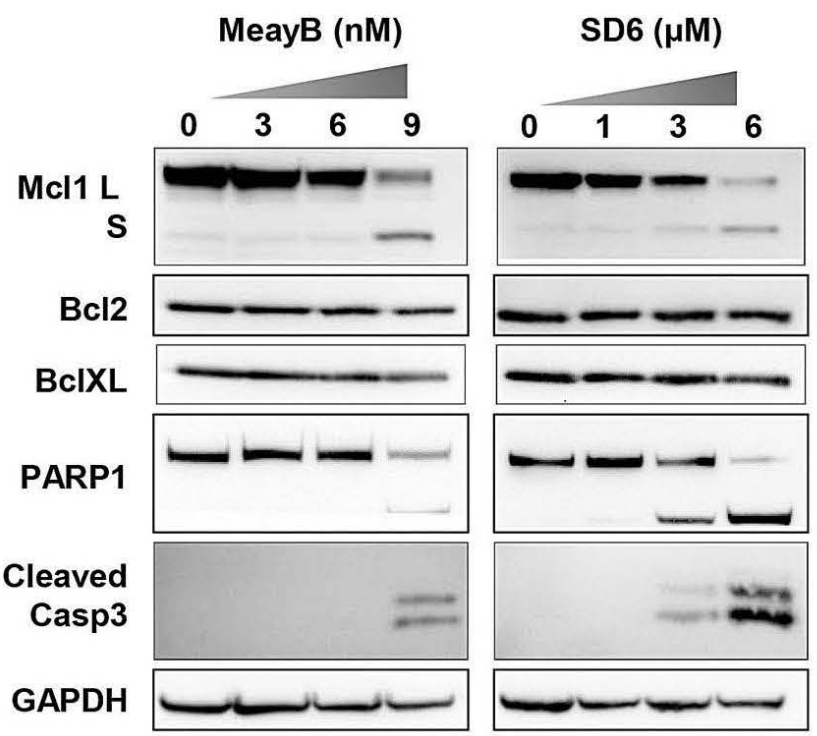

G

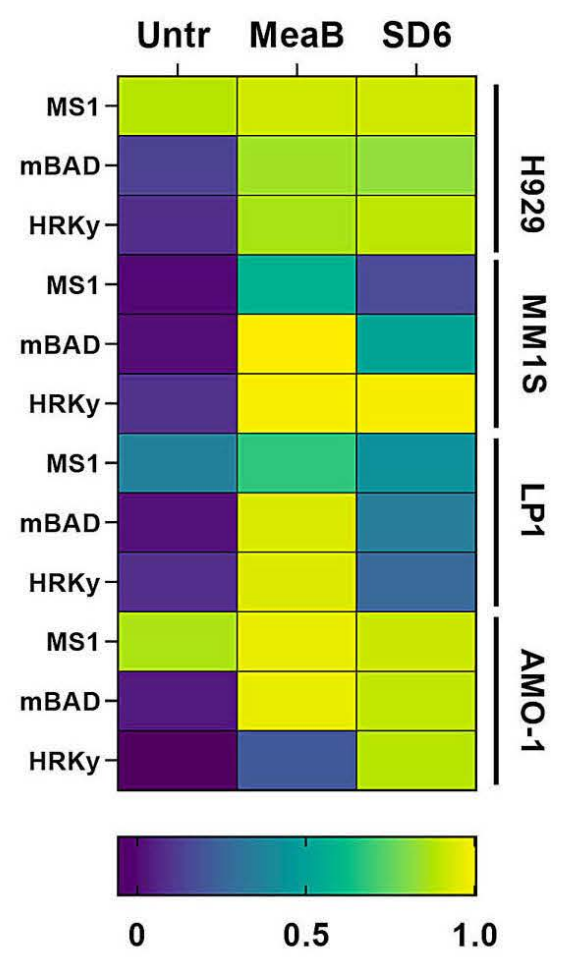

$\%$ Cytochrome c loss

Figure 4. BCL2 family member deregulation outlines splicing modulators activity on multiple myeloma cells. A) Pie chart showing the proportion of significant splice changes derived from ClariomD data of multiple myeloma (MM) cell lines treated with Meayamycin B compared with dimethyl sulfoxide (DMSO)-treated controls. Yellow slice indicates significant intron cassette exon events; purple slice indicates significant intron retention events; gray indicate all other complex categories of splice events. B) The top 10 pathways included in BioCarta gene sets enriched by fgsea R package among the 1,000 most significant mis-spliced genes in Meayamycin B-treated cells. C) Bubble plot shows the enrichment scores, $P$-values and the types of aberrant splicing event in the top mis-spliced genes of apoptosis pathway after Meayamycin B treatment compared with control cells. D and E) Reverse transcription polymerase chain reaction (RT-PCR) analyses of H929 cells after 6-hour treatment with growing doses of

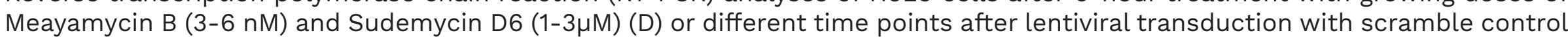
or SF3B1 specific shRNAs clone\#1 (E), to assess levels of MCL1 (L, long and S, short isoforms), BCLXL and BCL2. GAPDH is used as internal control. The length of the main amplified isoforms is indicated as base pairs (bp). F) Western blot analysis of MCL1, BCL2, BCLXL, PARP and cleaved caspase 3 protein expression upon treatment with Meayamycin B and SD6 in H929 cells. GAPDH was used as loading control. G) Heatmap of percentage Cytochrome c loss, as quantified by flow cytometry on indicated MM cell lines after 6-hour treatment with different chemicals as compared with DMSO-treated controls (purple, lowest value; yellow, highest value). After each treatment, MM cells were exposed to BH3 mimetic peptides (MS1 10 uM, mBAD and HRKy 100 uM for all cell lines except for MM1S where $50 \mu \mathrm{M}$ mBAD and HRKy were used) for 45 minutes at room temperature and subsequently stained for fluorescence activated cell sorting analysis. 
tolerated during the 2-weeks treatment (Online Supplementary Figure S14). The superior effect of the combination over single agents in reducing tumor volume and weight, (Figure 6A and B) was observed starting after 1 week of therapy. The decrease in tumor growth was associated with improved os of animals treated with the combination therapy, as compared with vehicle and single agents (Figure 6C, $P<0.05$ ). Remarkably, animals treated with Venetoclax monotherapy had a similar survival to that of vehicle-treated animals $(P=0.15)$, confirming the lack of sensitivity of this model to Venetoclax treatment alone. In line with this data, immunohistochemical analyses revealed a dramatic increase of cleaved caspase-3 positive MM cells, with marked reduction of mitotic percentage (Figure 6D and E). Additionally, increased apoptotic bodies were observed in tumors derived from mice treated with the Venetoclax combination (Figure 6E). We also evaluated in vivo efficacy of Venetoclax combination with the clinical grade SF3b-targeting splicing modulator E7107. ${ }^{34}$ Mice injected with human MM1S cells were treated with vehicle, E7107 (2.5 mg/kg intravenous, once a day for 5 days), Venetoclax (100 mg/kg oral administration, once a day for 5 days) and their combination. As observed in our previous model, E7107-treated tumors resulted in significant tumor regression compared to control mice and mice treated with Venetoclax alone and, the effect of E7107 was further enhanced by the combination with Venetoclax, as shown in Figure 6F $(P<0.05)$. The mean tumor volume of mice treated with combination was lower than single agent treated mice bearing tumors with higher activity at day 54. Improved outcome was also observed for the combination group of mice, with a median survival significantly longer compared to those treated with vehicle or monotherapies (combo vs. vehicle $P<0.05$; combo vs. Venetoclax $P<0.001$; combo vs. E7107 $P<0.05$ ) (Figure 6G). Overall, these data support the potential benefits of splicing inhibition to enhance anti-MM effects of Venetoclax.

\section{Discussion}

The process of RNA splicing regulates gene function in normal cells, but only recently its deregulation has been implicated in driving the development and/or maintenance of cancer. ${ }^{9,35}$ Indeed, comprehensive genomic analyses have revealed accumulation of transcriptome changes, including aberrantly spliced products during tumor progression and drug resistance occurrence, thus identifying spliceosome machinery deregulation as crucial for tumor cells growth and making its targeting an innovative strategy for successful anti-cancer approaches. ${ }^{9}$ In
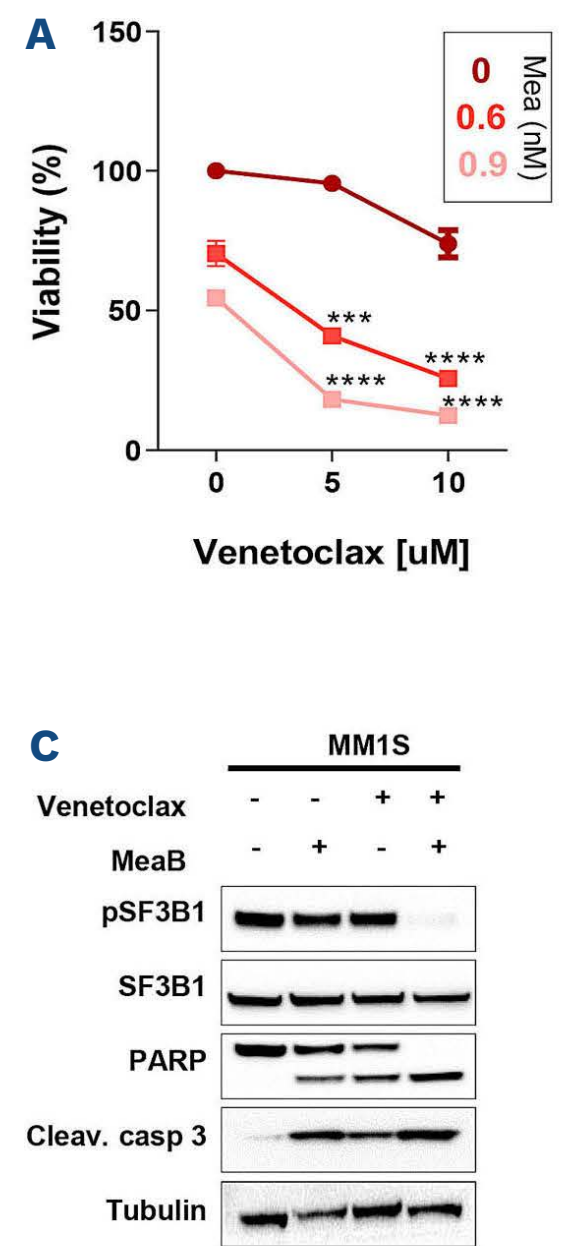
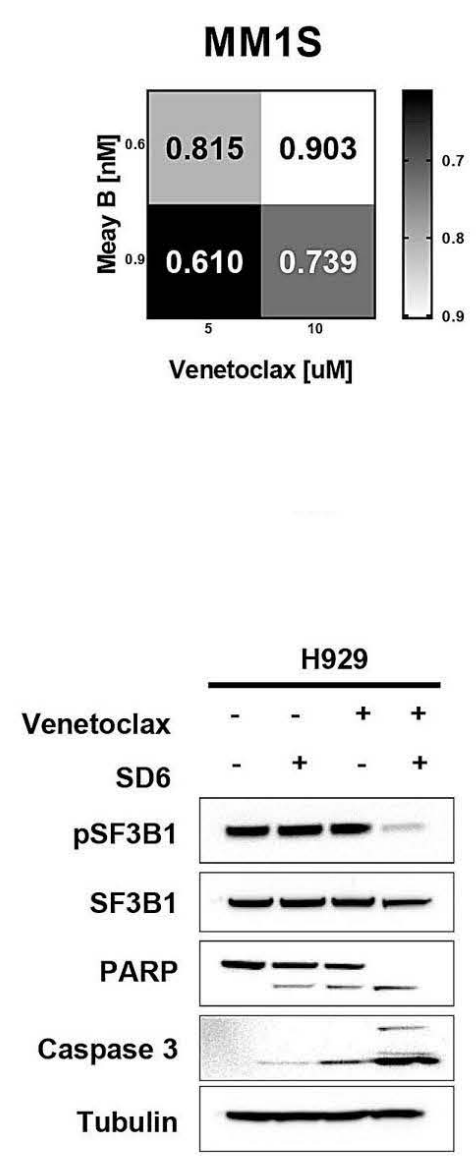
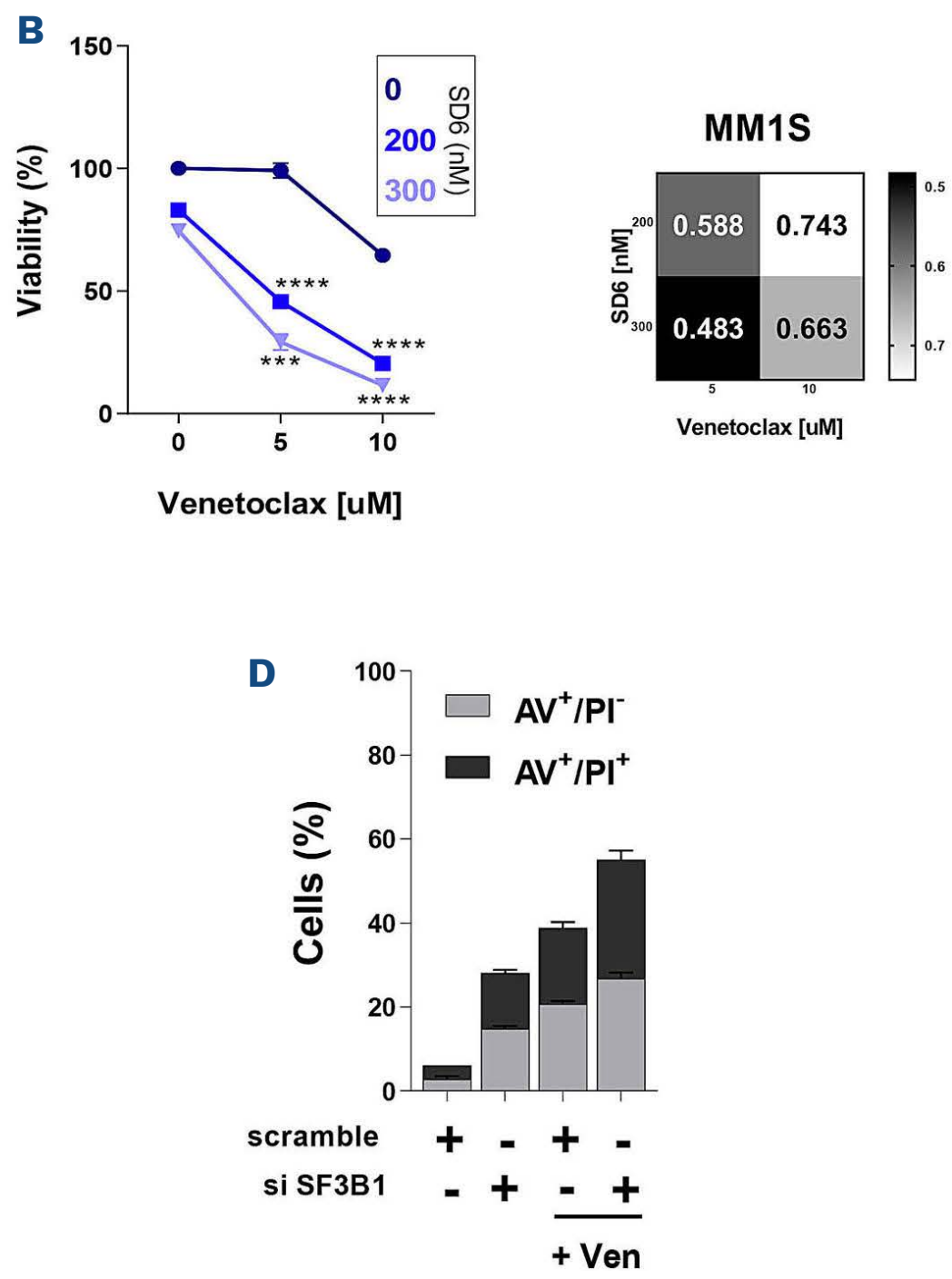

Figure 5. Continued on following page. 

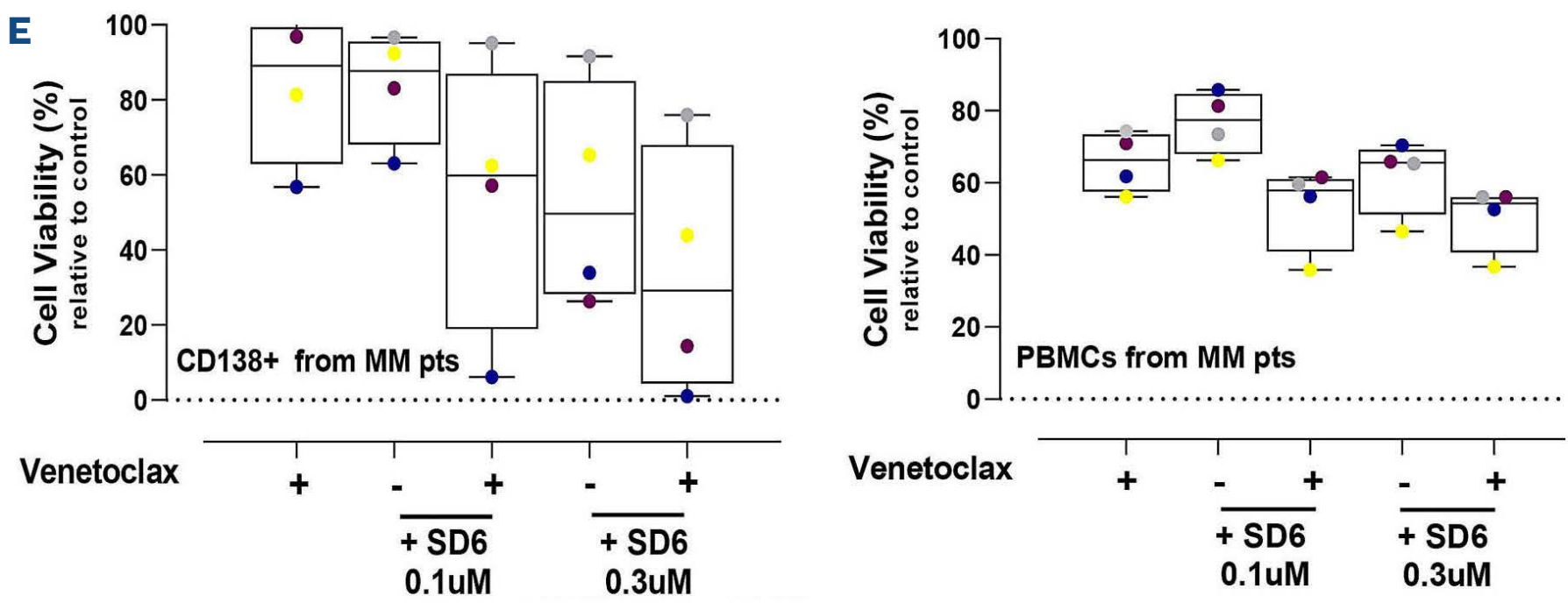
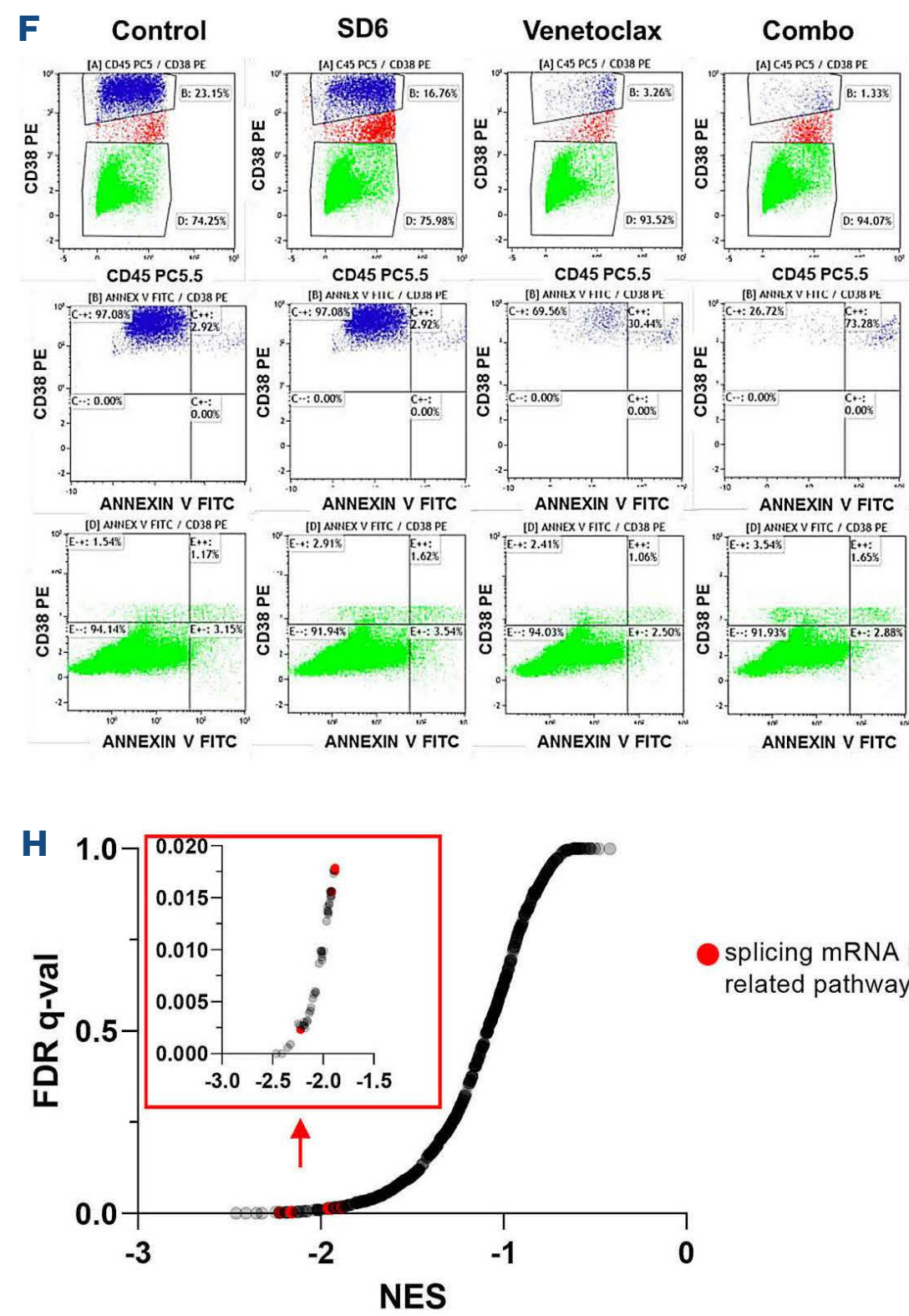

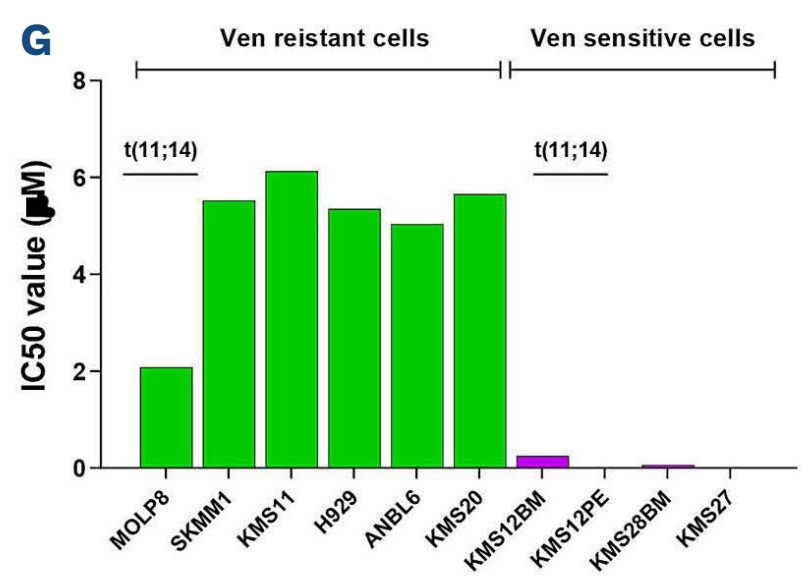

splicing mRNA processing related pathways

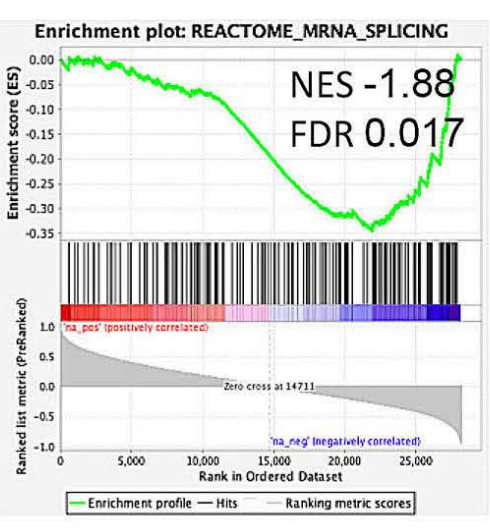

Figure 5. Splicing modulators sensitize multiple myeloma cells to Venetoclax by deregulating BCL2 family-members. Cell viability curves of multiple myeloma (MM) cells treated with combination therapies using Meayamycin B (A) or Sudemycin D6 (B) and Venetoclax. $\mathrm{Cl}$ synergy score (calculated with CalcuSyn software) for each set of drugs combination is indicated. Data are presented as mean \pm standard deviation (SD) ( $n=3)$. ( $* * * P \leq 0.001$, $* * * * P \leq 0.0001$; unpaired $t$-test). C) Immunoblots for phosphoSF3B1, SF3B1, PARP, caspase 3, and GAPDH on MM cell lines following each stimulus (indicated in figure) at 24 hours. D) Apoptotic cell death assessed with flow cytometry analysis after Annexin V/propidium iodide (PI) staining of AMO-1 cells SF3B1-silenced (nucleofected with specific small interfering RNA (siRNA) or control cells (siRNA scramble) treated with Venetoclax (7.5 $\mu$ M) for 48 hours. Displayed are data represented as mean $+/-$ SD in all $(n=3)$. E) CD138+ cells (left) and peripheral blood mononuclear cells (PBMC) (right) collected from MM patients were treated with indicated doses of SD6, Venetoclax (0.5 $\mu$ M) and their combination for 48 hours. Cell viability was measured by CTG assay. Cells deriving from the same patient are represented with same color in each graph. F) Flow plots of 1 representative MM patient sample. Corresponding sensitivity of MM-gated cells (CD38+/CD45-) and bone marrow stromal cells (BMSC) (CD38-/CD45-) to Venetoclax, SD6and cotreatment are shown. G) Indicated human multiple myeloma cell line $(\mathrm{HMCL})(\mathrm{n}=10)$ were treated with different doses of Venetoclax (Ven) for 24 hours and cell survival was assessed by CTG. Half maximal inhibitory concentration (IC ${ }_{50}$ ) analysis was performed with GraphPad software. $n=3$ independent experiments. H) Gene set enrichment analysis (GSEA) normalized enriched scores (NES) and falsediscovery rate (FDR) q-values for top enriched pathways, according to Reactome gene set, among de-regulated genes of Venetoclax resistant vs. sensitive cell lines; red square indicates the most significant region with splicing-related pathways accumulation (left); examples of GSEA-derived enrichment plots for genes involved in the splicing machinery (right). 
such a context, small molecules including spliceostatin, sudemycins, pladienolide and its derivatives are emerging as groundbreaking tools to fight cancers, especially those bearing splicing gene mutations. Since the RNA splicing apparatus is frequently affected by a wide range of driving events, including oncogenic MYC-activation and MCL1 addiction, a synthetic-lethal relationship between different tumor "Achille's heel" and core-spliceosome inhibition has been proposed, resulting in the increased use of splicing modulators for cancer treatment. 9,19,27,36

Here, we first confirmed the already reported upregulation of alternative splicing processes in MM cells by analyzing clinically annotated high throughput RNA sequencing data from the CoMMPass study. A pervasive splicing aberration was found in these cells to be associated with aberrant expression of RNA splicing machinery components and to significantly impact on overall clinical outcome. In line with these data, proliferating MM cells exhibited greater levels of spliceosome component SF3B1 than normal cells suggesting its role for tumor cell growth. Alternative splicing affects a wide range of genes with prevalent impairment of various cellular processes, including cell- cycle. Indeed, splicing isoforms have been reported for key cellcycle factors including cell division cycle 25 (CDC25), aurora kinase B (AURKB), CDC-Like kinase 1 (CLK1) and CDK2. ${ }^{37,38}$ Thus, tumor cell proliferation rate might be respon-

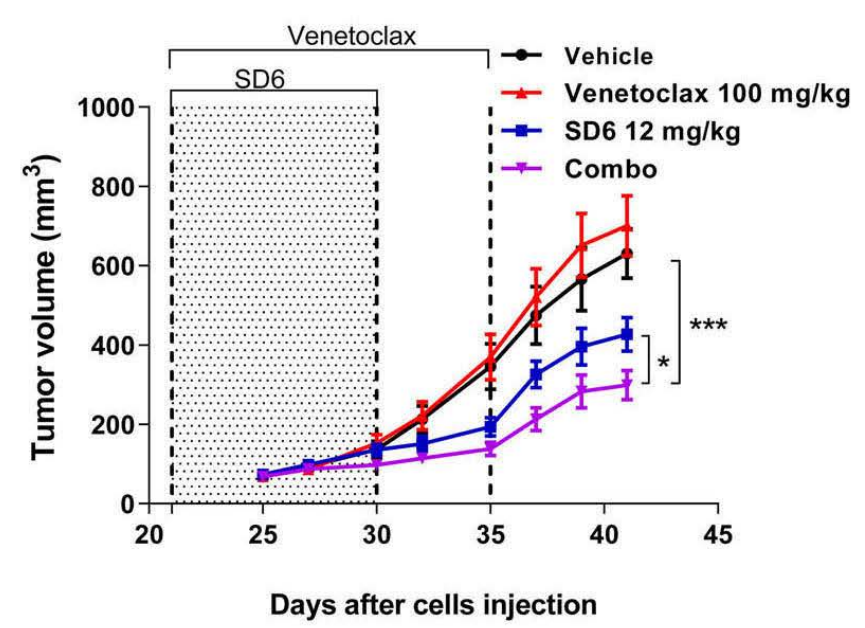

C

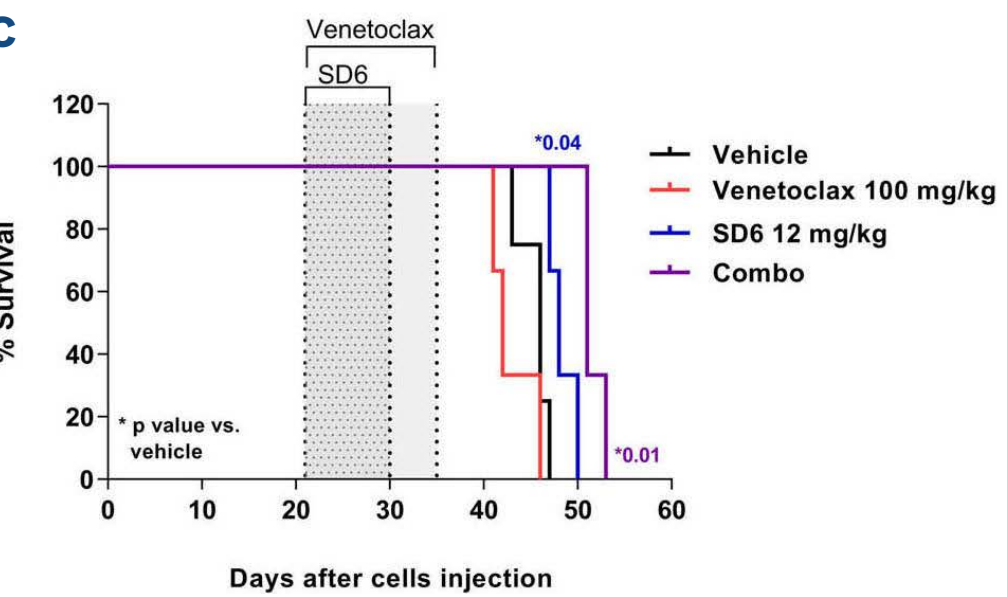

sible for high SF3B1 levels observed in these cells, but more investigations are needed. Despite the low mutational burden reported for splicing factors in MM, a striking anti-tumor effects of splicing modulators was observed to be associated with a significant change in MCL1 spliced isoforms in treated cells than specific control. These results are in accordance with other reports showing differential sensitivity to splicing modulation in BCL2 family genes, providing novel insights into mechanisms for spliceosome-targeted based approaches in tumors. ${ }^{29}$ The effect on MCL1 caused by SF3B1 targeting resulted in a cellular addiction shifting from MCL1 to BCL2 thus providing a rationale for combination of splicing modulators with the BCL2 inhibitor Venetoclax to achieving a synergistic activity against MM cell growth and viability. Indeed, a significant impact of this combination was observed on MM cells both in vitro and in vivo, while sparing non-cancerous cells thus providing evidence for a favorable therapeutic index.

Apoptosis is a highly regulated intracellular process, essential for normal cells survival and development; defects in regulation of this process result in a number of abnormalities, including cancer. ${ }^{39}$ Several B-cell malignancies such as chronic lymphocytic leukemia (CLL), lymphomas and plasma cell neoplasms, present different interaction between subgroups of the BCL2-family members includ-

B
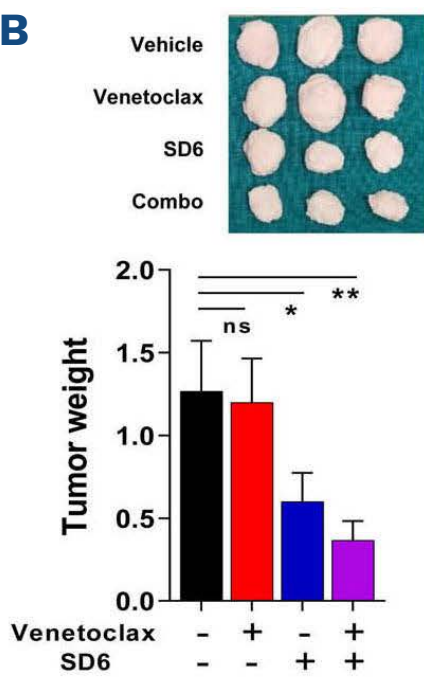

D
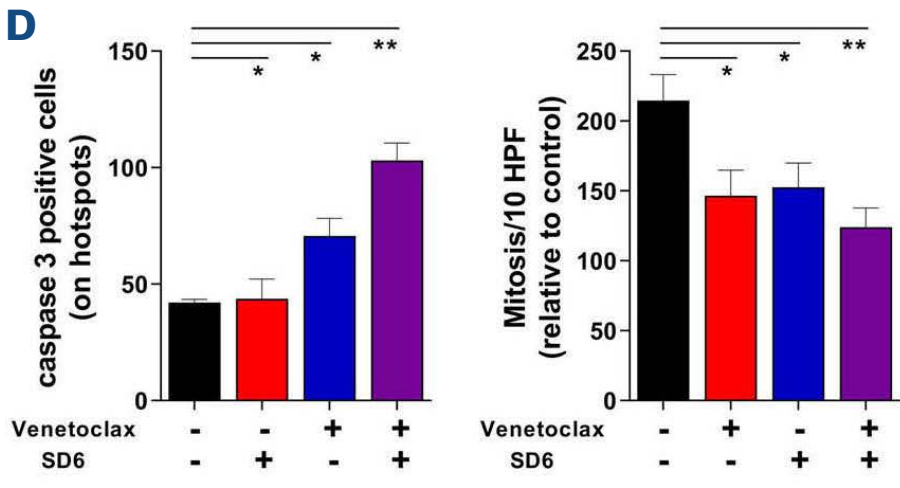

Figure 6. Continued on following page. 

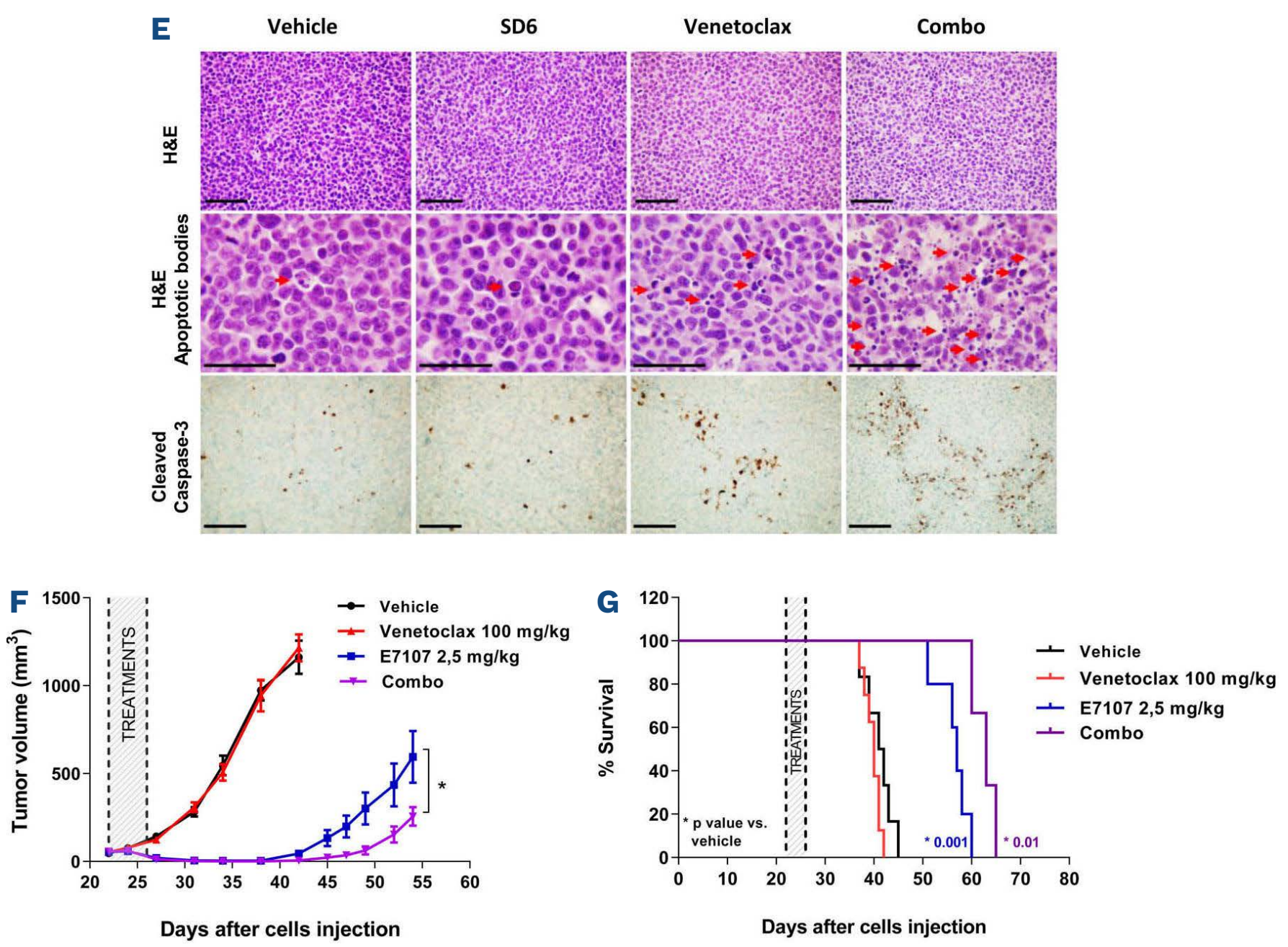

Figure 6. Splicing inhibitors synergize with Venetoclax in vivo in multiple myeloma xenograft murine models A) MM1S cells $\left(4,5 \times 10^{6}\right.$ cells/mouse) were implanted in both flanks of female NOD/SCID J mice (8 weeks of age). Tumor-bearing mice were randomized and treated with vehicle $(n=10)$; Venetoclax $(100 \mathrm{mg} / \mathrm{kg} ; \mathrm{n}=10)$ administered by oral gavage once a day for 15 days; SD6 (12 mg/kg; $n=10)$ intratumorally once a day for 10 days and their combination ( $\mathrm{n}=10)$. A significant delay in tumor growth in combination-treated mice was noted compared to vehicle-treated control mice ( $* * * p \leq 0.001$ ). Data are mean tumor volume \pm standard error of the mean (SEM). B) At the end of the experiment, mice were sacrificed, and tumor masses were imaged and weighed. (n.s.: not significant, ${ }^{*} P \leq 0.05, * * P \leq 0.01$ ). C) Kaplan-Meier analysis showing significant survival benefit for mice treated with the combo, compared with single-agent treatment SD6 ( $P=0.0169$, Log-rank Mantel-Cox test). D) Histogram pots show caspase 3-positive cells and mitosis observed in 10 observations in tumors harvested from mice treated with indicated stimuli. $(* P \leq 0.05, * * P \leq 0.01)$. E) Immunohistochemical analysis for hematoxylin and eosin (H\&E) and cleaved caspase 3 in xenografts tumors harvested from mice treated with control, SD6, Venetoclax or co-treatment. Tumor sections from treated and untreated mice stained with H\&E were also analyzed for apoptotic bodies formation by using higher magnification. Red arrows indicate apoptotic bodies in each panel. Scale bar, $50 \mu \mathrm{m}$. F) MM1S xenograft-bearing, 8-week-old female NOD/SCID J mice were treated with vehicle $(n=6)$; E7107 (2.5 mg/kg; $n=9)$ intravenous once a day for 5 days; Venetoclax (100 mg/kg; $n=8)$ oral administration once a day for 5 days and their combination $(n=11)$. A significant delay in tumor growth in co-treated mice was noted compared to E7107-treated control mice ( $\left.{ }^{*} P=0.0455\right)$. Bars indicate mean \pm SEM. G) Kaplan-Meier survival plot showing significant increase in survival of mice receiving the combination of E7107 plus Venetoclax compared to E7107 single agent-treated mice (combo vs. E7107 $P=0.0295) . \mathrm{n}$ indicates the number of tumors per treatment group. Data were analyzed by two-tailed Student's $t$-test (A, $B, D, F)$ or by Log-rank Mantel-Cox test $(C, G)$.

ing the pro-survival BCL2 (BCL2, MCL1, BCL2A1 and BCLXL) and the BH3-only proteins causing apoptosis. In these tumors, drug exposure results in perturbed intrinsic apoptotic pathway with mitochondrial apoptotic dependencies reprogramming and cell-death evasion. A proof-of-concept for this strategy is represented by the breakthrough or small molecules mimicking BH3-only proteins, which have totally changed the treatment landscape of CLL and acute myeloid leukemia. ${ }^{40}$ Also MM exhibit high dependence on apoptosis as suggested by efficacy of phase I clinical trial with Venetoclax in monotherapy (clinicaltrials gov. Identifier: NCT02755597) in RRMM patients, mainly in those harboring the $\mathrm{t}(11 ; 14)$ translocation. ${ }^{33}$ Unfortunately, changes in apoptosis regulators result in clinical resis- tance, thus supporting alternative strategies for these patients, including multiple apoptotic proteins inhibition. ${ }^{41,42}$ $40 \%$ of MM patients harbor gain or amplification status of $1 \mathrm{q} 21$ which makes these cells addicted to MCL1 and extremely vulnerable to its targeting, as suggested by preclinical studies. ${ }^{30,43}$ However, MCL1 has proven to be more challenging to target than BCL2, despite the long- standing interest in designing potent and selective MCL1 inhibitors for therapeutic use that have resulted in poor clinical benefits with few trials still ongoing (clinicaltrials gov. Identifier: NCT02992483, NCT02675452 and NCT03465540). We demonstrate here that modulation of splicing by selectively targeting MCL1, results in enhanced BCL2 activity on MM cells, and supports the synergistic 
activity observed in combination with Venetoclax. Remarkably, no significant effects were observed on nontumor cells derived from the same MM patient and treatment was well tolerated in mice as well, suggesting a safe therapeutic window of tested combinations. Emerging studies suggest that tumor cells, including MM, hijack spliceosome activity to evade anti-tumor therapies: mis-spliced forms of binding protein CRBN and broadscale intron retention have been observed in $\mathrm{MM}$ patients with immunomodulatory drugs or protease inhibitor resistance occurrence, respectively. ${ }^{16,44}$ Thus, splicing interference represents an exploitable vulnerability to overcome drug resistance but still deserves further investigations. Here, we confirm that alternative splicing is largely perturbed in MM cells and constitutes an attractive source for novel therapeutic strategies. Low dose of splicing modulators, by shifting apoptotic dependencies, results in enhanced $\mathrm{BH} 3$ mimetic Venetoclax susceptibility, irrespective of specific genomic landscapes including splicing factor-mutations, c-MYC-expression or $\mathrm{t}(11 ; 14)$ status, providing rationale for the use of this combination for the treatment of all MM patients.

\section{Disclosures}

No conflicts of interest to disclose.

\section{Contributions}

$D S$ and $M C$ designed the research, performed experiments, analyzed the data and wrote the manuscript; $C M, P B, E G$, $S R, P C$ and $D R$ performed experiments and analyzed the data; $P B$ performed mice experiments; $L M$ performed IHC analyses; $A C, A L, F G, M M, P M, S A, R V, M F$ and AlN provided patient samples; MP performed immunofluorescence analysis; AnN, KT, MS, MF and NM performed genomic analyses; MC and RML revised the final version of manuscript.

\section{Acknowledgments}

The authors thank Dr. Jeremy Ryan and Prof. Anthony Letai for $B H 3$ profiling experiments setup. They acknowledge also the MMRF for the access to CoMMpass study data.

\section{Funding}

This work was supported in part by the Associazione Italiana per la Ricerca sul Cancro (AIRC, MYFG \#18491, IG \#2328 to MC and MYFG \# 21552 to AC), Italian Ministry of Health (GR-2016-02361523 to AC, and $5 \times 1000$ funds 2016 to MC) NIH grant P01-155258-07 (to MF, MKS, and NCM), Associazione Italiana Leucemie Linfomi e Mieloma (AIL sezione di Genova) and University of Genoa, Genoa, Italy.

\section{References}

1. Palumbo A, Anderson K. Multiple myeloma. N Engl J Med. 2011;364(11):1046-1060.

2. Kumar SK, Rajkumar V, Kyle RA, et al. Multiple myeloma. Nat Rev Dis Prim. 2017;3(1):17046.

3. Leung-Hagesteijn C, Erdmann N, Cheung G, et al. Xbp1sNegative tumor $\mathrm{B}$ cells and pre-plasmablasts mediate therapeutic proteasome inhibitor resistance in multiple myeloma. Cancer Cell. 2013;24(3):289-304.

4. Cea M, Cagnetta A, Adamia S, et al. Evidence for a role of the histone deacetylase SIRT6 in DNA damage response of multiple myeloma cells. Blood. 2016;127(9):1138-1150.

5. Soncini D, Minetto P, Martinuzzi C, et al. Amino acid depletion triggered by L-asparaginase sensitizes MM cells to carfilzomib by inducing mitochondria ROS-mediated cell death. Blood Adv. 2020;4(18):4312-4326.

6. Ast G. How did alternative splicing evolve? Nat Rev Genet. 2004;5(10):773-782.

7. Graubert TA, Shen D, Ding L, et al. Recurrent mutations in the U2AF1 splicing factor in myelodysplastic syndromes. Nat Genet. 2012;44(1):53-57.

8. Krämer A. The structure and function of proteins involved in mammalian pre-mRNA splicing. Annu Rev Biochem. 1996;65:367-409.

9. Lee SCW, Abdel-Wahab O. Therapeutic targeting of splicing in cancer. Nat Med. 2016;22(9):976-986.

10. Webb TR, Joyner AS, Potter PM. The development and application of small molecule modulators of SF $3 \mathrm{~b}$ as therapeutic agents for cancer. Drug Discov Today. 2013;18(12):43-49.
11. Yoshida K, Sanada M, Shiraishi Y, et al. Frequent pathway mutations of splicing machinery in myelodysplasia. Nature. 2011;478(7367):64-69.

12. Quesada V, Conde L, Villamor N, et al. Exome sequencing identifies recurrent mutations of the splicing factor SF3B1 gene in chronic lymphocytic leukemia. Nat Genet. 2012;44(1):47-52.

13. Maguire SL, Leonidou A, Wai P, et al. SF3B1 mutations constitute a novel therapeutic target in breast cancer. $J$ Pathol. 2015;235(4):571-580.

14. Bauer MA, Ashby C, Wardell C, et al. Differential RNA splicing as a potentially important driver mechanism in multiple myeloma. Haematologica. 2021;106(3):736-745.

15. Walker BA, Mavrommatis K, Wardell CP, et al. Identification of novel mutational drivers reveals oncogene dependencies in multiple myeloma. Blood. 2018;132(6):587-597.

16. Huang HH, Ferguson ID, Thornton AM, et al. Proteasome inhibitor-induced modulation reveals the spliceosome as a specific therapeutic vulnerability in multiple myeloma. Nat Commun. 2020;11(1):1931.

17. Ryan J. A Guide to BH3 Profiling METHOD 2: iBH3 PROFILING. 2017.

18. Adamia S, Bar-Natan M, Haibe-Kains B, et al. NOTCH2 and FLT3 gene mis-splicings are common events in patients with acute myeloid leukemia (AML): new potential targets in AML. Blood. 2014;123(18):2816-2825.

19. Koh CM, Bezzi M, Low DHPP, et al. MYC regulates the core premRNA splicing machinery as an essential step in lymphomagenesis. Nature. 2015;523(7558):96-100.

20. Adamia S, Abiatari I, Amin SB, et al. The effects of MicroRNA 
deregulation on pre-RNA processing network in multiple myeloma. Leukemia. 2020;34(1):167-179.

21. Zamani-Ahmadmahmudi M, Dabiri S, Nadimi N. Identification of pathway-based prognostic gene signatures in patients with multiple myeloma. Transl Res. 2017;185:47-57.

22. Laganà $A$, Perumal $D$, Melnekoff $D$, et al. Integrative network analysis identifies novel drivers of pathogenesis and progression in newly diagnosed multiple myeloma. Leukemia. 2018;32(1):120-130.

23. Kotake $\mathrm{Y}$, Sagane K, Owa T, et al. Splicing factor SF3b as a target of the antitumor natural product pladienolide. Nat Chem Biol. 2007;3(9):570-575.

24. Kaida D, Motoyoshi H, Tashiro E, et al. Spliceostatin A targets $\mathrm{SF} 3 \mathrm{~b}$ and inhibits both splicing and nuclear retention of premRNA. Nat Chem Biol. 2007;3(9):576-583.

25. Folco EG, Coil KE, Reed R. The anti-tumor drug E7107 reveals an essential role for SF3b in remodeling U2 snRNP to expose the branch point-binding region. Genes Dev. 2011;25(5):440-444.

26. Marchesini M, Ogoti Y, Fiorini E, et al. ILF2 Is a Regulator of RNA splicing and DNA damage response in 1q21-amplified multiple myeloma. Cancer Cell. 2017;32(1):88-100.

27. Hsu TYT, Simon LM, Neill NJ, et al. The spliceosome is a therapeutic vulnerability in MYC-driven cancer. Nature. 2015;525(7569):384-388.

28. Certo M, Moore VDG, Nishino M, et al. Mitochondria primed by death signals determine cellular addiction to antiapoptotic BCL-2 family members. Cancer Cell. 2006;9(5):351-365.

29. Ruefli-Brasse A, Reed JC. Therapeutics targeting Bcl-2 in hematological malignancies. Biochem J. 2017;474(21):3643-3657.

30. Kotschy A, Szlavik Z, Murray J, et al. The MCL1 inhibitor 563845 is tolerable and effective in diverse cancer models. Nature. 2016;538(7626):477-482.

31. Moujalled DM, Pomilio G, Ghiurau C, et al. Combining BH3mimetics to target both BCL-2 and MCL1 has potent activity in pre-clinical models of acute myeloid leukemia. Leukemia. 2019;33(4):905-917.

32. Prukova D, Andera L, Nahacka Z, et al. Cotargeting of BCL2 with venetoclax and MCL1 with $\mathrm{S} 63845$ is synthetically lethal in vivo in relapsed mantle cell lymphoma. Clin Cancer Res. 2019;25(14):4455-4465.
33. Kumar S, Kaufman JL, Gasparetto C, et al. Efficacy of venetoclax as targeted therapy for relapsed/refractory $t(11 ; 14)$ multiple myeloma. Blood. 2017;130(22):2401-2409.

34. Hong DS, Kurzrock R, Naing A, et al. A phase I, open-label, single-arm, dose-escalation study of E7107, a precursor messenger ribonucleic acid (pre-mRNA) splicesome inhibitor administered intravenously on days 1 and 8 every 21 days to patients with solid tumors. Invest New Drugs. 2014;32(3):436444.

35. Chen S, Benbarche S, Abdel-Wahab O. Splicing factor mutations in hematologic malignancies. Blood. 2021;138(8):599-612.

36. Aird D, Teng T, Huang C-L, et al. Sensitivity to splicing modulation of BCL2 family genes defines cancer therapeutic strategies for splicing modulators. Nat Commun. 2019;10(1):137.

37. Petasny M, Bentata M, Pawellek A, et al. Splicing to keep cycling: the Importance of pre-mRNA splicing during the cell cycle. Trends Genet. 2021;37(3):266-278.

38. Jorge J, Petronilho S, Alves R, et al. Apoptosis induction and cell cycle arrest of pladienolide $B$ in erythroleukemia cell lines. Invest New Drugs. 2020;38(2):369-377.

39. Hanahan D, Weinberg RA. The hallmarks of cancer. Cell. 2000;100(1):57-70.

40. DiNardo CD, Jonas BA, Pullarkat V, et al. Azacitidine and Venetoclax in previously untreated acute myeloid leukemia. $\mathrm{N}$ Engl J Med. 2020;383(7):617-629.

41. van Delft MF, Wei AH, Mason KD, et al. The BH3 mimetic ABT737 targets selective $\mathrm{Bcl}-2$ proteins and efficiently induces apoptosis via Bak/Bax if Mcl-1 is neutralized. Cancer Cell. 2006;10(5):389-399.

42. Gupta VA, Barwick BG, Matulis SM, et al. Venetoclax sensitivity in multiple myeloma is associated with $B$ cell gene expression. Blood. 2021;137(26):3604-3615.

43. Slomp A, Moesbergen LM, Gong JN, et al. Multiple myeloma with 1q21 amplification is highly sensitive to MCL-1 targeting. Blood Adv. 2019;3(24):4202-4214.

44. Gooding S, Ansari-Pour N, Towfic F, et al. Multiple cereblon genetic changes are associated with acquired resistance to lenalidomide or pomalidomide in multiple myeloma. Blood. 2021;137(2):232-237. 